J. DIFFERENTIAL GEOMETRY

45 (1997) 349-389

\title{
ON THE INTEGRABLE GEOMETRY OF SOLITON EQUATIONS AND N=2 SUPERSYMMETRIC GAUGE THEORIES
}

\author{
I. M. KRICHEVER \& D. H. PHONG
}

\begin{abstract}
We provide a unified construction of the symplectic forms which arise in the solution of both $\mathrm{N}=2$ supersymmetric Yang-Mills theories and soliton equations. Their phase spaces are Jacobian-type bundles over the leaves of a foliation in a universal configuration space. On one hand, imbedded into finite-gap solutions of soliton equations, these symplectic forms assume explicit expressions in terms of the auxiliary Lax pair, expressions which generalize the well-known Gardner-Faddeev-Zakharov bracket for KdV to a vast class of $2 \mathrm{D}$ integrable models; on the other hand, they determine completely the effective Lagrangian and BPS spectrum when the leaves are identified with the moduli space of vacua of an $\mathrm{N}=2$ supersymmetric gauge theory. For $\mathrm{SU}\left(N_{c}\right)$ with $N_{f} \leq N_{c}+1$ flavors, the spectral curves we obtain this way agree with the ones derived by Hanany and $\mathrm{Oz}$ and others from physical considerations.
\end{abstract}

\section{Introduction}

A particularly striking aspect of the recent solutions of $\mathrm{N}=2 \mathrm{su}-$ persymmetric Yang-Mills theories [1] -[4] has been the emergence of integrable structures [5] - [6], structures which had surfaced in the completely different context of soliton equations and their Whithamaveraged counterparts [7], [9], [10]. On the gauge theory side, the moduli space of inequivalent vacua is identified with a moduli space of certain compact Riemann surfaces $\Gamma$, and both the effective Lagrangian and the Bogomolny-Prasad-Sommerfeld spectrum can be read off from the periods of a meromorphic 1 -form $\mathrm{d} \lambda$ on $\Gamma$. A defining property of $\mathrm{d} \lambda$ is that its external derivative $\delta \mathrm{d} \lambda$ be a holomorphic symplectic form $\omega$

Received May 9, 1996. Work supported in part by the National Science Foundation under grant DMS-95-05399 
on the total space of the bundle whose fiber is the Jacobian $J(\Gamma)$ of $\Gamma$. On the soliton side, $\Gamma$ coincides with the spectral curve of certain Toda or spin chain equations [5], [11], and $\mathrm{d} \lambda$ with the pre-potential of their Whitham equations [10]. Moreover, the symplectic form $\omega$ coincides with the symplectic structure of these chains, considered as finite-dimensional Hamiltonian systems.

The most general type of finite-dimensional integrable system in soliton theory is a space of algebraic-geometric, or finite-gap, solutions to a soliton equation [12]. In their work in the early 1980 's, Novikov and Veselov [13] had proposed a notion of analytic symplectic form and a Hamiltonian theory for algebraic-geometric solutions connected with hyperelliptic curves. Most of the symplectic forms $\omega$ which have arisen so far in supersymmetric gauge theories are special cases of the Novikov-Veselov forms. As shown in the original 1994 Seiberg-Witten [1] work on $\mathrm{SU}(2)$ gauge theories with hypermultiplets in the fundamental representation, and in the subsequent Donagi-Witten work [6] on the $\mathrm{SU}(2)$ theory with matter in the adjoint representation, the symplectic forms are also the true indicators of integrability, and often suffice to identify the spectral curves themselves.

The main goal of this paper is to build more systematically the common foundations of the above two theories. We construct general algebraic-geometric symplectic forms defined on phase spaces $\mathcal{N}^{g}$ which are Jacobian-type bundles over the leaves $\mathcal{M}$ of a foliation on a universal configuration space. This last space is a fundamental ingredient of our approach, and is defined itself as the moduli space of all algebraic curves with a fixed pair of Abelian integrals (c.f. Section II).

Our construction of symplectic forms and their phase spaces is entirely geometric. To make contact with integrable models, the phase space $\mathcal{N}^{g}$ for each leaf is imbedded in a corresponding space of functions as a moduli space of algebraic-geometric solutions to the soliton equation. The algebraic-geometric symplectic form $\omega_{\mathcal{M}}$ is identified with the restriction to $\mathcal{N}^{g}$ of a symplectic form defined on the space of functions. Remarkably, in terms of the auxiliary Lax pair of the soliton equation, these symplectic forms assume completely explicit and simple expressions.

As an example, consider a two-dimensional soliton equation with the flat curvature representation

$$
\left[\partial_{y}-L, \partial_{t}-A\right]=0 \leftrightarrow L_{t}-A_{y}+[L, A]=0,
$$


where

$$
L=\sum_{i=1}^{n} u_{i}(x, y, t) \partial_{x}^{i}, \quad A=\sum_{i=1}^{m} v_{i}(x, y, t) \partial_{x}^{i}
$$

are linear operators with $(N \times N)$ matrix coefficients. Then the symplectic form $\omega_{\mathcal{M}}$ is given by (c.f. Section IV)

$$
\omega_{\mathcal{M}}=-\sum_{\alpha=1}^{N} \operatorname{Res}_{P_{\alpha}} \frac{\left\langle\delta \psi^{+} \wedge\left(L^{(1)} \delta A-A^{(1)} \delta L\right) \psi>\right.}{\left\langle\psi^{+} \psi>\right.} \mathrm{d} p
$$

where $P_{\alpha}$ are punctures on the surface, $\psi, \psi^{+}$are the Baker-Akhiezer and dual Baker-Akhiezer functions, $L^{(1)}, A^{(1)}$ are the first descendants of the operators $L$ and $A$ :

$$
L^{(1)}=\sum_{i=1}^{n} i u_{i}(x, y, t) \partial_{x}^{i-1}, A^{(1)}=\sum_{i=1}^{m} i v_{i}(x, y, t) \partial_{x}^{i-1},
$$

and $\mathrm{d} p$ is the differential of the quasi-momentum for the Baker-Akhiezer function. The formula (1) can be rewritten in turn in terms of the $\delta u_{i}$ 's. In this form, they unify many of the known symplectic forms for spatially 1D models, including e.g. the Gardner-Faddeev-Zakharov form $\left\langle\delta u \wedge \int^{x} \delta u>\right.$ familiar from KdV. More importantly, they also provide seemingly new symplectic structures for many 1D models, as well as a vast generalization to a whole variety of $2 \mathrm{D}$ integrable models for which no symplectic form had been available so far.

There are strong indications that the above universal configuration space can also serve as a universal space for the effective Lagrangians of $N=2$ supersymmetric gauge theories. Indeed, it suffices to imbed the moduli space of vacua of the gauge theory as a leaf in the universal configuration space, in order to obtain the desired Seiberg-Witten fibration as a pull-back of the existing fibration. Geometric considerations suggest a natural imbedding in the case of $\mathrm{SU}\left(N_{c}\right)$ Yang-Mills theories with $N_{f} \leq 2 N_{c}$ flavors. It is intriguing that this imbedding reproduces the known solutions of [1] - [3] when $N_{f} \leq N_{c}+1$, but diverges from the formulas conjectured by Hanany and $\mathrm{Oz}$ [3] for $N_{c}+2 \leq N_{f} \leq 2 N_{c}$. A central issue here is a proper identification of coordinates for the universal configuration space with the order parameters of the Yang-Mills theories. This can only be resolved by a more careful analysis of the monodromies of the resulting effective action at weak coupling. We shall return to this issue elsewhere. 


\section{The universal configuration space}

We shall construct the universal configuration space as a moduli space of Riemann surfaces $\Gamma$ with $N$ punctures $\left(P_{\alpha}\right)_{\alpha=1}^{N}$, and two Abelian integrals $E$ and $Q$ with poles of orders at most $n=\left(n_{\alpha}\right)_{\alpha=1}^{N}, m=$ $\left(m_{\alpha}\right)_{\alpha=1}^{N}$ at the punctures. In view of the subtleties inherent to the multiple-valuedness of Abelian integrals, it is convenient to proceed as follows.

We define an $n_{\alpha}$-jet $\left[z_{\alpha}\right]_{n_{\alpha}}$ of coordinates near a puncture $P_{\alpha}$ to be an equivalence class of coordinates $z_{\alpha}$ near $P_{\alpha}$, with $z_{\alpha}$ and $z_{\alpha}^{\prime}$ equivalent if $z_{\alpha}^{\prime}=z_{\alpha}+O\left(z_{\alpha}^{n_{\alpha}+1}\right)$. Evidently, the space of $n_{\alpha}$-jets of coordinates near $P_{\alpha}$ has finite dimension, equal in fact to $n_{\alpha}$. Henceforth, we let $P_{1}$ be a marked puncture. In presence of a jet $[z]_{n}$ near $P_{1}$, we can define an Abelian integral $Q$ as a pair $\left(\mathrm{d} Q, c_{Q}\right)$, where $\mathrm{d} Q$ is a meromorphic differential (or Abelian differential) on the surface $\Gamma$, and

$$
Q=\sum_{k=-m}^{\infty} c_{k} z^{k}+c_{Q}+R^{Q} \log z
$$

if $\mathrm{d} Q=\mathrm{d}\left(\sum_{k=-m}^{\infty} c_{k} z^{k}\right)+R^{Q} \frac{d z}{z}$ is the expansion of $\mathrm{d} Q$ near $P_{1}$. By integrating $\mathrm{d} Q$ along paths, we can then extend the Abelian integral $E$ holomorphically to a neighborhood of any point in $\Gamma \backslash\left\{P_{1}, \cdots, P_{N}\right\}$. The analytic continuation will depend in general on the path, and we also keep track of its homotopy class.

Fix now the multi-indices $n=\left(n_{1}, \cdots, n_{N}\right)$ and $m=\left(m_{1}, \cdots, m_{N}\right)$. The universal configuration space $\mathcal{M}_{g}(n, m)$ can then be defined as

$$
\mathcal{M}_{g}(n, m)=\left\{\Gamma, P_{\alpha},\left[z_{\alpha}\right]_{n_{\alpha}} ; E, Q\right\}
$$

where $\Gamma$ is a smooth genus $g$ Riemann surface with $N$ ordered points $P_{\alpha}$, $\left[z_{\alpha}\right]_{n_{\alpha}}$ is an $n_{\alpha}$-jet of coordinates near each $P_{\alpha}$, and $E, Q$ are Abelian integrals with the following expansions near the punctures $P_{\alpha}$

$$
\begin{aligned}
E & =z_{1}^{-n_{1}}+c_{E}+R_{1}^{E} \log z_{1}+O\left(z_{1}\right), \\
\mathrm{d} E & =\mathrm{d}\left(z^{-n_{\alpha}}+O\left(z_{\alpha}\right)\right)+R_{\alpha}^{E} \frac{\mathrm{d} z_{\alpha}}{z_{\alpha}}, \\
Q & =\sum_{k=1}^{m_{1}} c_{1, k} z_{1}^{-k}+c_{Q}+R_{1}^{Q} \log z_{1}+O\left(z_{\alpha}\right), \\
\mathrm{d} Q & =\mathrm{d}\left(\sum_{k=1}^{m_{\alpha}} c_{\alpha, k} z_{\alpha}^{-k}+O\left(z_{\alpha}\right)\right)+R_{\alpha}^{Q} \frac{\mathrm{d} z_{\alpha}}{z_{\alpha}} .
\end{aligned}
$$


The space $\mathcal{M}_{g}(n, m)$ is a complex manifold with only orbifold singularities, and its complex dimension is equal to

$$
\operatorname{dim} \mathcal{M}_{g}(n, m)=5 g-3+3 N+\sum_{\alpha=1}^{N}\left(n_{\alpha}+m_{\alpha}\right)
$$

for $g>1$. Indeed, $3 g-3+N$ parameters account for the moduli space of genus $g$ Riemann surfaces with $N$ punctures, $\sum_{\alpha=1}^{N} n_{\alpha}$ parameters for the jets of coordinates $\left[z_{\alpha}\right]_{n_{\alpha}}, N+g$ parameters for the Abelian integral $E(N-1)$ parameters for the independent residues since their sum is 0,1 parameter for the constant $c_{E}$, and $g$ parameters for the Abelian differentials which can be added without modifying the singular expansions of (4)), and finally $N+g+\sum_{\alpha=1}^{N} m_{\alpha}$ for the Abelian integral $Q$. Alternatively, the number of degrees of freedom of an Abelian integral $E$ with poles of order $n=\left(n_{\alpha}\right)$ is $1+\sum_{\alpha=1}^{N}\left(n_{\alpha}+1\right)-1+g=N+g+\sum_{\alpha=1}^{N} n_{\alpha}$, where the first 1 corresponds to the additive constant, and the remaining integer on the left is the dimension of meromorphic differentials with poles of order $\leq n_{\alpha}+1$ at each $P_{\alpha}$. Together with a similar counting for $Q$ and the dimension of the moduli space of Riemann surfaces with punctures, we recover (4). Note that (4) gives the right dimension for $g=0,1 ; N>0$, although the counting of the dimension is slightly different.

We can introduce explicit local coordinates on $\mathcal{M}_{g}(n, m)$ (for their relation to the Whitham theory, see [10]). The first of these consists of the residues of the differentials $\mathrm{d} E$ and $\mathrm{d} Q$ :

$$
R_{\alpha}^{E}=\operatorname{Res}_{P_{\alpha}} \mathrm{d} E, R_{\alpha}^{Q}=\operatorname{Res}_{P_{\alpha}} \mathrm{d} Q, \alpha=2, \cdots, N .
$$

The next set of coordinates is only local on the universal configuration space, and requires some choices. First, we cut apart the Riemann surface $\Gamma$ along a homology basis $A_{i}, B_{j}, i, j=1, \cdots, g$, with the canonical intersection matrix $A_{i} \cdot A_{j}=B_{i} \cdot B_{j}=0, A_{i} \cdot B_{j}=\delta_{i j}$. By selecting cuts from $P_{1}$ to $P_{\alpha}$ for each $2 \leq \alpha \leq N$, we obtain a well-defined branch of the Abelian integrals $E$ and $Q$. Locally on the universal configuration space, this construction can be carried out continuously, with paths homotopic by deformations not crossing any of the poles. We consider first the case where $n_{\alpha}$ is at least 1 . Then there exists a unique local coordinate $z_{\alpha}$ in the jet $\left[z_{\alpha}\right]_{n_{\alpha}}$ such that

$$
E=z_{\alpha}^{-n_{\alpha}}+R_{\alpha}^{E} \log z_{\alpha}
$$


in the neighborhood of $P_{\alpha}$. We then set

$$
\begin{aligned}
& T_{\alpha, k}=\frac{1}{k} \operatorname{Res}_{P_{\alpha}}\left(z_{\alpha}^{k} Q \mathrm{~d} E\right), \alpha=1, \cdots, N, k=1, \cdots, n_{\alpha}+m_{\alpha}, \\
& T_{\alpha, 0}=\operatorname{Res}_{P_{\alpha}}(Q \mathrm{~d} E), \alpha=2, \cdots, N .
\end{aligned}
$$

When $n_{\alpha}=0$ and $R_{\alpha}^{E} \neq 0$, we choose the coordinate $z_{\alpha}$ by demanding that

$$
E-E\left(P_{\alpha}\right)=R_{\alpha}^{E} \log z_{\alpha} .
$$

A final set of coordinates for the universal configuration space can now be defined by

$$
\begin{aligned}
\tau_{A_{i}, E} & =\oint_{A_{i}} \mathrm{~d} E, \tau_{B_{i}, E}=\oint_{B_{i}} \mathrm{~d} E, \\
\tau_{A_{i}, Q} & =\oint_{A_{i}} \mathrm{~d} Q, \tau_{B_{i}, Q}=\oint_{B_{i}} \mathrm{~d} Q, \\
a_{i} & =\oint_{A_{i}} Q \mathrm{~d} E, i=1, \cdots, g .
\end{aligned}
$$

Let $\mathcal{D}$ be the open set in $\mathcal{M}_{g}(m, n)$ where the zero divisors of $\mathrm{d} E$ and $\mathrm{d} Q$, namely the sets $\{\gamma ; \mathrm{d} E(\gamma)=0\}$ and $\{\gamma ; \mathrm{d} Q(\gamma)=0\}$, do not intersect. Then we have

Theorem 1. (a) Near each point in $\mathcal{D}$, the

$$
5 g-3+3 N+\sum_{\alpha=1}^{N}\left(n_{\alpha}+m_{\alpha}\right)
$$

functions $R_{\alpha}^{E}, R_{\alpha}^{Q}, T_{\alpha, k}, \tau_{A_{i}, E}, \tau_{B_{i}, E}, \tau_{A_{i}, Q}, \tau_{B_{i}, Q}, a_{i}$ of (6)-(10) have linearly independent differentials, and thus define a local holomorphic coordinate system for $\mathcal{M}_{g}(n, m)$; (b) The joint level sets of the functions (6)-(9) define a smooth $g$-dimensional foliation of $\mathcal{D}$, independent of the choices we made to define the coordinates themselves.

To lighten the exposition of the paper, we postpone the proof of Theorem 1 to the Appendix.

The universal configuration space $\mathcal{M}_{g}(n, m)$ is the base space for a hierarchy of fibrations $\mathcal{N}_{g}^{k}(n, m)$ of particular interest to us. These are 
the fibrations whose fiber above each point of $\mathcal{M}_{g}(n, m)$ is the $k$-th symmetric power $S^{k}(\Gamma)$ of the curve $\Gamma$. The first, $\mathcal{N}_{g}^{k=1}(n, m) \equiv \mathcal{N}_{g}(n, m)$, is just a version of the universal curve, where the fiber above a point of $\mathcal{M}_{g}(n, m)$ is the Riemann surface $\Gamma$ itself. The fibration $\mathcal{N}_{g}^{g}(n, m)$ with $k=g$ can be viewed as a universal Jacobian, by identifying $S^{g}(\Gamma)$ with the Jacobian $J(\Gamma)$ via the Abel map

$$
\left(\gamma_{1}, \cdots, \gamma_{g}\right) \rightarrow \phi_{j}=\sum_{i=1}^{g} \int_{P_{1}}^{\gamma_{i}} \mathrm{~d} \omega_{j} .
$$

The fibrations with $k>g$ enter the study of matrix solitons, as we shall see later.

Consider now a leaf of the foliation described in Theorem 1 , denoted just by $\mathcal{M}$ for simplicity, and let $\mathcal{N}$ and $\mathcal{N}^{g}$ denote the above fibrations restricted to $\mathcal{M}$. To define a symplectic form $\omega_{\mathcal{M}}$ on $\mathcal{N}^{g}$, we begin by discussing some aspects of differentials of the Abelian integrals $E$ and $Q$ on $\mathcal{N}$.

The first key observation is that, although the Abelian integrals $E$ and $Q$ are multi-valued functions on the universal fibration of curves over the moduli space $\mathcal{M}_{g}(n, m)$, their differentials are well-defined on $\mathcal{N}$. Indeed, by our normalizations, $E$ and $Q$ are well-defined in a small neighborhood of the puncture $P_{1}$. Their analytic continuations by different paths can only change by multiples of their residues or periods along closed cycles. Since along a leaf of the foliation, these ambiguities remain constant, they disappear upon differentiation. We shall denote these differentials along the fibrations by $\delta E$ and $\delta Q$. Acting on vectors tangent to the fiber, they reduce of course to the usual differentials $\mathrm{d} E$ and $\mathrm{d} Q$.

Next, we note that the choice of an Abelian integral, say $E$, also provides us with a meromorphic connection $\nabla^{E}$ on $\mathcal{N}$. Indeed, at any point of $\mathcal{N}$, the variety $E=$ constant is intrinsic and transversal to the fiber. This means we can differentiate functions on $\mathcal{N}$ along vector fields $X$ on $\mathcal{M}$, simply by lifting these vector fields to vector fields tangent to the varieties $E=$ constant. More generally, we can differentiate 1-forms $\mathrm{d} f$ on the fibration of curves $\Gamma$ by setting

$$
\nabla_{X}^{E}(\mathrm{~d} f)=\nabla_{X}^{E}\left(\frac{\mathrm{d} f}{\mathrm{~d} E}\right) \mathrm{d} E .
$$

Furthermore, the differential $\mathrm{d} Q$ of any Abelian integral $Q$ along the fiber can be extended to a 1 -form on the whole manifold $\mathcal{N}$ by making it zero along $E=$ constant. We still denote this differential by 
$\mathrm{d} Q$. Equivalently, we can trivialize the fibration $\mathcal{N}$ with the variables $a_{1}, \cdots, a_{g}$ along the leaf $\mathcal{M}$, and the variable $E$ along the fiber. The form $\mathrm{d} Q$ which we defined previously coincides with $\frac{\mathrm{d} Q}{\mathrm{~d} E} \mathrm{~d} E$, where $\mathrm{d} E$ is viewed as one of the elements of the basis of 1 -forms for $\mathcal{N}$ in this coordinate system. The full differential $\delta Q$ is given by

$$
\delta Q=\mathrm{d} Q+\sum_{i=1}^{g} \frac{\partial Q}{\partial a_{i}} d a_{i} \equiv \mathrm{d} Q+\delta^{E} Q .
$$

For $E$, we have $\delta E=\mathrm{d} E$, and the above connection reduces to $\nabla_{\partial_{a_{i}}}^{E}=$ $\partial_{a_{i}}$.

If we consider now the full differential $\delta(Q \mathrm{~d} E)$ on $\mathcal{N}$, it is readily seen that it is well-defined, despite the multivaluedness of $Q$. In fact, the partial derivatives $\partial_{a_{i}}(Q \mathrm{~d} E)$ along the base $\mathcal{M}$ are holomorphic, since the singular parts of the differentials as well as the ambiguities are all fixed. In particular,

$$
\frac{\partial}{\partial a_{i}}(Q \mathrm{~d} E)=\mathrm{d} \omega_{i}
$$

where $d \omega_{i}$ is the basis of normalized holomorphic differentials

$$
\oint_{A_{i}} \mathrm{~d} \omega_{j}=\delta_{i j}, \oint_{B_{i}} \mathrm{~d} \omega_{j}=\tau_{i j}
$$

and $\tau_{i j}$ the period matrix of $\Gamma$. We can now define the desired symplectic form $\omega_{\mathcal{M}}$ on $\mathcal{N}^{g}$ by

$$
\omega_{\mathcal{M}}=\delta\left(\sum_{i=1}^{g} Q\left(\gamma_{i}\right) \mathrm{d} E\left(\gamma_{i}\right)\right)=\sum_{i=1}^{g} \delta Q\left(\gamma_{i}\right) \wedge \mathrm{d} E\left(\gamma_{i}\right)=\sum_{i=1}^{g} \mathrm{~d} a_{i} \wedge \mathrm{d} \omega_{i}
$$

In many situations, we need to go beyond the case $\mathcal{N}^{g}$, and consider the fibration $\mathcal{N}^{k}$ for $k>g$. The above form on the leaves $\mathcal{M}$ is then degenerate. However, a non-degenerate form for $k>g$ can be obtained by restricting $\mathcal{N}^{k}$ to the larger leaves $\tilde{\mathcal{M}}$ of the fibration corresponding to the level sets of all the functions (6)-(9) except for $T_{\alpha, 0}$. Thus we can set

$$
\omega_{\tilde{\mathcal{M}}}=\sum_{i=1}^{k} \delta Q\left(\gamma_{i}\right) \wedge \mathrm{d} E\left(\gamma_{i}\right)=\sum_{i=1}^{g} \mathrm{~d} a_{i} \wedge \mathrm{d} \omega_{i}+\sum_{\alpha=2}^{k-g+1} \mathrm{~d} T_{\alpha, 0} \wedge \widetilde{\mathrm{d} \omega_{\alpha}},
$$


where $\widetilde{\mathrm{d} \omega_{\alpha}}$ is a normalized differential with only simple poles at $P_{1}$ and $P_{\alpha}$, and residues -1 and 1 respectively. This form $\omega_{\tilde{\mathcal{M}}}$ is meromorphic. So far we do not know its role, if any, in supersymmetric gauge theories, but it is responsible for the symplectic structure of finite-gap solutions to integrable equations with matrix Lax operators (see Section IV).

\section{The symplectic form for the KP hierarchy}

Remarkably, the natural geometric symplectic form constructed in the last section leads directly to a Hamiltonian structure for soliton equations. We shall derive it explicitly in the case of the KadomtsevPetviashvili (or KP) hierarchy, and show that it reduces, in the case of $\mathrm{KdV}$, to the familiar Gardner-Faddeev-Zakharov symplectic form on the space of functions of one variable.

\section{1) Solutions of the KP Hierarchy}

We begin by recalling some notions from the algebraic-geometric theory of solitons [12]. A fundamental principle is that to each data consisting of a smooth Riemann surface $\Gamma$ with $N$ punctures $P_{\alpha}$, local holomorphic coordinates $z_{\alpha}$ around each puncture, and a divisor $\left(\gamma_{1}, \cdots, \gamma_{g}\right)$, there corresponds a sequence $\left\{u_{i, n}^{\alpha}(t)\right\}_{1 \leq i \leq n-2,2 \leq n<\infty}$ of complex, quasi-periodic functions of an arbitrarily large number of variables $t=\left(t_{n, \alpha}\right)_{n=1}^{\infty}$, which are solutions of an infinite integrable basic hierarchy of soliton equations. The $\left\{u_{i, n}^{\alpha}\right\}$ are obtained via the BakerAkhiezer function $\psi(t, z), z \in \Gamma$, which is the unique function meromorphic away from $P_{\alpha}$, with simple poles at $\gamma_{i}, i=1, \cdots, g$, and which admits the following essential singularity at $P_{\alpha}$

$$
\psi\left(t, z_{\alpha}\right)=\exp \left(\sum_{n=1}^{\infty} t_{n, \alpha} z_{\alpha}^{-n}\right) \sum_{i=0}^{\infty} \xi_{\alpha, i}(t) z_{\alpha}^{i}, \quad \xi_{1,0}=1 .
$$

For each $n$, it is then straightforward to derive recursively a unique linear differential operator in $\partial / \partial t_{1, \alpha}$

$$
L_{n}^{\alpha}=\left(\frac{\partial}{\partial t_{1, \alpha}}\right)^{n}+\sum_{i=1}^{n-1} u_{i, n}^{\alpha}\left(\frac{\partial}{\partial t_{1, \alpha}}\right)^{i},
$$

so that

$$
\left(\frac{\partial}{\partial t_{n, \alpha}}-L_{n}^{\alpha}\right) \psi(t, z)=0 .
$$


The coefficients of $L_{n}$ are the functions we seek, and the soliton equations they satisfy are the partial differential equations in the variables $t$ resulting from the commutation relations $\left[\frac{\partial}{\partial t_{n, \alpha}}-L_{n}^{\alpha}, \frac{\partial}{\partial t_{m, \alpha}}-L_{m}^{\alpha}\right]=0$. If we let

$$
\widehat{\mathcal{N}}^{k}=\left\{\Gamma, P_{\alpha}, z_{\alpha}, \gamma_{1}, \cdots, \gamma_{k}\right\}
$$

be the space of data of the above form, we have in this way a map from $\widehat{\mathcal{N}}^{g}$ to the space of sequences of functions $\left\{u_{i, n}^{\alpha}(t)\right\}$. The space $\hat{\mathcal{N}}^{k}$ is of course infinite-dimensional, but we shall soon restrict to a more manageable finite-dimensional subspace. The above-defined Baker-Akhiezer functions correspond to linear operators with scalar coefficients. In the general case, the Baker-Akhiezer vector-functions which are defined by (15) with $k=g+l-1$ give solutions to the integrable hierarchy with the flat curvature representation and linear operators with matrix $(l \times l)$ coefficients (see section IV).

The type of basic hierarchy is characterized by $N$ and $k$. For example, $N=1, k=g$ give the KP hierarchy, while $N=2, k=g$ give the 2D Toda lattice hierarchy. The KdV hierarchy is a reduction of the KP hierarchy, and in fact, all known hierarchies are reductions of the basic ones. Henceforth, we shall restrict our attention to the KP hierarchy, where $N=1$, and simply drop the index $\alpha$ from our notation.

It is instructive to write the Baker-Akhiezer function more explicitly. The key ingredients are the meromorphic differentials $\mathrm{d} \Omega_{n}$, which are characterized by their expansion $\mathrm{d} \Omega_{n}=\mathrm{d}\left(z^{-n}+O(z)\right)$ near $P$, and by their normalization

$$
\operatorname{Re} \oint_{C} \mathrm{~d} \Omega_{n}=0
$$

for any cycle $C$. The corresponding Abelian integrals $\Omega_{n}$ can then be defined as

$$
\Omega_{n}=z^{-n}+O(z)
$$

near the puncture $P$. As before, $\Omega_{n}$ can therefore be extended by analytic continuation to a neighborhood of an arbitrary point in the Riemann surface $\Gamma$. Its value depends on the homotopy class of the path along which the analytic continuation is performed, and we keep track of this path as well. The Baker-Akhiezer function can thus be expressed 
as $[9]$

$$
\psi(t, z)=\exp \left(\sum_{n=1}^{\infty} t_{n} \Omega_{n}\right) \Phi(t, z)
$$

with the quasi-periodic function $\Phi(t, z)$ given by

$$
\begin{aligned}
\Phi(t, z)= & \frac{\Theta\left(\int^{z} \omega_{i}-\sum_{j=1}^{g} \int^{\gamma_{j}} \omega_{i}+\sum_{t=n}^{\infty} \frac{t_{n}}{2 \pi_{i}}\left(\oint_{A_{i}} \mathrm{~d} \Omega_{n}-\sum_{j=1}^{g} \tau_{i j} \oint_{B_{j}} \mathrm{~d} \Omega_{n}\right)+K\right)}{\Theta\left(\int^{P} \omega_{i}-\sum_{j=1}^{g} \int^{\gamma_{j}} \omega_{i}+\sum_{t=n}^{\infty} \frac{t_{n}}{2 \pi_{i}}\left(\oint_{A_{i}} \mathrm{~d} \Omega_{n}-\sum_{j=1}^{g} \tau_{i j} \oint_{B_{j}} \mathrm{~d} \Omega_{n}\right)+K\right)} \\
& \times \frac{\Theta\left(\int^{P} \omega_{i}-\sum_{j=1}^{g} \int^{\gamma_{j}} \omega_{i}+K\right)}{\Theta\left(\int^{z} \omega_{i}-\sum_{j=1}^{g} \int^{\gamma_{j}} \omega_{i}+K\right)} \exp \left(-\sum_{n=1}^{\infty} t_{n} \sum_{j=1}^{g} \oint_{B_{j}} \mathrm{~d} \Omega_{n} \int_{P}^{z} \omega_{j}\right),
\end{aligned}
$$

where $\tau_{i j}$ is the period matrix of $\Gamma$, and $K$ is the vector of Riemann constants. We note that although the Abelian integrals $\Omega_{n}$ as well as the Abel map $\int^{z} \omega_{i}$ are path dependent, this dependence cancels in the full expression for the Baker-Akhiezer function, as it should.

For later use, we also recall here the main properties of the dual Baker-Akhiezer function $\psi^{+}(t, z)$. To define it, we note that the RiemannRoch theorem implies that for $g$ points $\gamma_{1}, \cdots, \gamma_{g}$ in general position, the unique meromorphic differential

$$
\mathrm{d} \Omega=\mathrm{d}\left(z^{-1}+\sum_{s=2}^{\infty} a_{s} z^{s}\right)
$$

with double pole at $P$ and zeroes at $\gamma_{1}, \cdots, \gamma_{g}$, must also have $g$ other zeroes. We denote these by $\gamma_{1}^{+}, \cdots, \gamma_{g}^{+}$. The dual Baker-Akhiezer function $\psi^{+}(t, z)$ is then the unique function $\psi^{+}(t, z)$ which is meromorphic everywhere except at $P$, has at most simple poles at $\gamma_{1}^{+}, \cdots, \gamma_{g}^{+}$, and admits the following expansion near $P$

$$
\psi^{+}(t, z)=\exp \left(-\sum_{n=1}^{\infty} t_{n} z^{-n}\right)\left(1+\sum_{s=1}^{\infty} \xi_{s}^{+}(t) z^{s}\right) .
$$

It is then not difficult to check that the dual Baker-Akhiezer function satisfies the equation

$$
-\frac{\partial}{\partial t_{n}} \psi^{+}(t, z)=\psi^{+}(t, z) L_{n}
$$

where the operator $L_{n}$ is the same as the one of (14), and the above left (adjoint) action of differential operators is defined by

$$
\left(f^{+} \partial_{t_{1}}^{i}\right)=(-1)^{i}\left(\partial_{t_{1}}^{i} f^{+}\right)
$$


The coefficients $\xi_{s}^{+}$of the expansion (19) for the dual Baker-Akhiezer function are differential polynomials in the coefficients $\xi_{s}$ of the BakerAkhiezer function. In fact, we have

$$
\operatorname{Res}_{P} \psi^{+}(t, z)\left(\partial_{t_{1}}^{m} \psi(t, z)\right) \mathrm{d} \Omega=0
$$

since the differential on the left-hand side is meromorphic everywhere, and holomorphic away from $P$. This implies that

$$
\sum_{l=0}^{m} \sum_{s+i+j=1} C_{m}^{l} a_{s} \xi_{i}^{+}\left(\partial_{t_{1}}^{m-l} \xi_{j+l}\right)=0,
$$

where $a_{0}=1, a_{1}=0$, and $a_{s}$ are the coefficients of the expansion (18) of $\mathrm{d} \Omega$ near $P$. These equations determine $\xi_{s}^{+}$recursively through $\xi_{s}$. For example,

$$
\xi_{1}^{+}=-\xi_{1}, \xi_{2}^{+}=-\xi_{2}+\xi_{1}^{2}-\partial_{t_{1}} \xi_{1} .
$$

2) The imbedding of the leaves $\mathcal{M}$ into the space of KP solutions

Consider now the fibration $\mathcal{N}_{g}^{g}(n, 1)$ of the last section, in the case of a single $(N=1)$ puncture $P$. Recall that its base $\mathcal{M}_{g}(m, 1)$ consists of Riemann surfaces $\Gamma$ with a puncture $P$, of jets $[z]_{n}$ of coordinates around $P$, and of Abelian integrals $E, Q$ with only poles at $P$ of order $n$ and 1 respectively. Its fiber over each point is simply the $g$-symmetric power of $\Gamma$. The basic observation is that an element in $\mathcal{N}_{g}^{g}(n, 1)$ gives rise to a data in $\hat{\mathcal{N}}^{g}$. Indeed, as we already noted when introducing local coordinates for $\mathcal{M}_{g}(n, m)$, the Abelian integral $E$ characterizes a unique holomorphic coordinate around $P$ in the given jet $[z]_{n}$, satisfying $E(z)=z^{-n}$ and the fact that it is in the given jet. We obtain in this way a map

$$
\left(\Gamma, P,[z]_{n}, E, Q ; \gamma_{1}, \cdots, \gamma_{g}\right) \rightarrow\left(\Gamma, P, z ; \gamma_{1}, \cdots \gamma_{g}\right)
$$

from $\mathcal{N}_{g}^{g}(n, 1)$ into the space of data $\widehat{\mathcal{N}}^{g}$, and hence into the space of algebraic-geometric solutions of the KP hierarchy

$$
\mathcal{N}_{g, 1}^{g} \rightarrow\left\{\left(u_{i, n}(t)\right)_{i=1}^{n-2}\right\} .
$$

We restrict our attention now to a real leaf $\mathcal{M}$, that is, a leaf of the foliation of Theorem 1, which satisfies the following additional condition

$$
\operatorname{Re} \oint_{C} \mathrm{~d} E=\operatorname{Re} \oint_{C} \mathrm{~d} Q=0
$$


for all cycles $C$ on the punctured surface $\Gamma$. We say then that the differentials $\mathrm{d} E$ and $\mathrm{d} Q$ are real-normalized. This condition is unambiguous. On real leaves, the Abelian differentials $\mathrm{d} E$ and $\mathrm{d} Q$ are readily recognized as the Abelian differentials $\mathrm{d} Q=\mathrm{d} \Omega_{1}$ and $\mathrm{d} E=\mathrm{d} \Omega_{n}$ of (17), simply by comparing their singularities at $P$.

Our main goal is to express the symplectic form $\omega_{\mathcal{M}}$ which we constructed earlier in terms of forms on the space of functions $\left\{u_{i, n}(t)\right\}$. The functions $u_{i, n}(t)$ can be written explicitly in terms of the coefficients $\xi_{s}$ of the Baker-Akhiezer function $\psi(t, z)$ with the help of the equations

$$
\sum_{i=0}^{n} u_{i, n} \sum_{l=0}^{i} C_{i}^{l}\left(\partial_{x}^{i-l} \xi_{s+l}\right)=\xi_{s+n}, \quad s=-n+2, \ldots,-1,0 .
$$

They are differential polynomials in the first $n-1$ coefficients $\xi_{s}(t)$ of the expansion (13) of $\psi$.

The equations for $u_{i, n}$ are almost invertible. Let us consider them as a system of equations for unknown functions $\xi_{s}, s=1, \ldots, n-1$ with $u_{i, n}$ given. Then $\xi_{s}, s \leq n-1$ are uniquely defined by this system if we fix the initial data:

$$
\left.\xi_{s}(t)\right|_{x=0}=\varphi_{s}\left(t_{2}, t_{3}, \ldots\right) .
$$

That ambiguity does not effect the first $n-1$ coefficients of an expansion of the logarithmic derivative of $\psi$.

The $n-1$ leading coefficients $h_{1}(t), \cdots, h_{n-1}(t)$ of the expansion

$$
\frac{\partial_{t_{1}} \psi}{\psi}=z^{-1}+\sum_{s=1}^{\infty} h_{s} z^{s}
$$

are differential polynomials

$$
h_{s}(t)=h_{s}\left(u_{i, n}, \partial_{t_{1}} u_{i, n}, \cdots\right)
$$

in the $u_{i, n}$ 's. The same is true for the first $n-1$ coefficients $h_{1}^{+}(t), \cdots, h_{n-1}^{+}(t)$ of the expansion of the dual Baker-Akhiezer function

$$
\frac{\partial_{t_{1}} \psi^{+}}{\psi^{+}}=-z^{-1}+\sum_{s=1}^{\infty} h_{s}^{+} z^{s}
$$


The differential polynomials $h_{s}$ and $h_{s}^{+}$are universal and depend on $n$ only. For the remainder of this section as well as Section IV, we shall adopt a notation of greater use in the study of soliton equations, and set

$$
x=t_{1}, p=\Omega_{1}
$$

as the basic space-variable and its corresponding quasi-momentum. We also fix an $n$, and set $y=t_{n}$. In this notation, we have for example

$$
\begin{aligned}
h_{1}= & -\frac{1}{n} u_{n-2, n}, \\
h_{2}= & \frac{n-1}{n} u_{n-2, n ; x}-\frac{1}{n} u_{n-3, n}, \\
h_{3}= & -\frac{(n-1)(n-2)}{6 n} u_{n-2, n ; x}+\frac{n+1}{2 n^{2}} u_{n-2, n}^{2} \\
& +\frac{n-1}{2} u_{n-3, n ; x}-\frac{1}{n} u_{n-4, n},
\end{aligned}
$$

while the first few coefficients $h_{s}^{+}$are given by

$$
\begin{aligned}
h_{1}^{+} & =\frac{1}{n} u_{n-2, n}, \\
h_{2}^{+} & =\frac{3-n}{2 n} u_{n-2, n ; x}+\frac{1}{n} u_{n-3, n} .
\end{aligned}
$$

The mean values of the polynomials $h_{s}(t)$ are equal to the first coefficients of the expansion of the Abelian integral $p=\Omega_{1}$ :

$$
\begin{aligned}
p & =z^{-1}+\sum_{s=1}^{\infty} H_{s} z^{s}, \\
H_{s} & =<h_{s}(t)>_{x} \equiv \lim _{L \rightarrow \infty} \int_{-L}^{L} \frac{d x}{2 L} h_{s}(x, y) .
\end{aligned}
$$

The bundles $\mathcal{N}^{g}$ over real leaves $\mathcal{M}$ of the foliated manifold $\mathcal{M}_{g}(n, 1)$ can now be recognized as the preimage under the map (21) of the level sets of the integrals $H_{s}=<h_{s}>_{x}$.

We can now state one of our main results:

Theorem 2. Let $\mathcal{N}^{g}$ be the Jacobian bundle over a real leaf $\mathcal{M}$ of the moduli space $\mathcal{M}_{g}(n, 1)$. Then the symplectic form $\omega_{\mathcal{M}}$ of (12) can be expressed as

$$
\omega_{\mathcal{M}}=\operatorname{Res}_{P} \frac{\left\langle\delta \psi^{+} \wedge \delta L \psi\right\rangle}{\left\langle\psi^{+} \psi\right\rangle} \mathrm{d} p=n \sum_{s=1}^{n-2}\left\langle\delta h_{s} \wedge \int^{t_{1}} \delta^{*} h_{n-s}^{+}\right\rangle
$$


where $h_{s}=h_{s}\left(u_{i, n}, \cdots\right)$ and $h_{s}^{+}\left(u_{i, n}, \cdots\right)$ are the above differential polynomials, the 1-forms $\delta h_{s}$ and $\delta^{*} h_{s}^{+}$are defined respectively by

$$
\delta h_{s}=\sum_{i=0}^{n-2} \delta u_{i, n} \sum_{l}\left(-\partial_{t_{1}}\right)^{l} \frac{\partial h_{s}}{\partial u_{i, n}^{(l)}}, \delta^{*} h_{s}^{+}=\sum_{i=0}^{n-2} \delta u_{i, n}^{(l)} \sum_{l} \frac{\partial h_{s}^{+}}{\partial u_{i, n}^{(l)}}
$$

and $\langle\cdot\rangle$ denotes the average with respect to both variables $x=t_{1}$ and $y=t_{n}$ of quasi-periodic functions

$$
<f>=\lim _{L, M \rightarrow \infty} \int_{-L}^{L} \frac{d x}{2 L} \int_{-M}^{M} \frac{\mathrm{d} y}{2 M} f(x, y) .
$$

Important Remark. In general, finite-gap solutions to soliton equations are complex meromorphic functions of all variables. This requires a more delicate definition of averaging. Without discussing this point in detail, we would like to mention that the averaging we adopt in this paper is valid at least for real and smooth solutions of soliton equations. The corresponding constraints for algebraic-geometric data singles out the real part of the configuration space.

Proof of Theorem 2. We had noted earlier that the full differentials of two Abelian differentials $\delta p$ and $\delta E$ are well-defined on the fibration $\mathcal{N}$. In terms of the imbedding (21), the differentials of $E=\Omega_{n}$ and $p=\Omega_{1}$ satisfy the following important relation [9]

$$
\delta E=\delta p \frac{\mathrm{d} E}{\mathrm{~d} p}+\frac{\left.<\psi^{+} \delta L_{n} \psi\right\rangle}{\left\langle\psi^{+} \psi\right\rangle}
$$

where $\delta L_{n}$ is given by

$$
\delta L_{n}=\sum_{i=0}^{n-2} \delta u_{i, n}(t) \partial_{x}^{i}
$$

Now the symplectic form $\omega_{\mathcal{M}}$ can be expressed as

$$
\omega_{\mathcal{M}}=\sum_{s=1}^{g} \delta p\left(\gamma_{s}\right) \wedge \mathrm{d} E\left(\gamma_{s}\right)
$$


As we had seen in the discussion leading to the equation (12), $\delta E=\mathrm{d} E$. Thus we may write in view of (31)

$$
\begin{aligned}
\omega_{\mathcal{M}} & =\sum_{s=1}^{g}\left(\mathrm{~d} E\left(\gamma_{s}\right)-\frac{\left\langle\psi^{+} \delta L_{n} \psi\right\rangle}{\left\langle\psi^{+} \psi\right\rangle}\left(\gamma_{s}\right)\right) \frac{\mathrm{d} p}{\mathrm{~d} E}\left(\gamma_{s}\right) \wedge \mathrm{d} E\left(\gamma_{s}\right) \\
& =-\sum_{s=1}^{g} \frac{\left\langle\psi^{+} \delta L \psi\right\rangle}{\left\langle\psi^{+} \psi\right\rangle}\left(\gamma_{s}\right) \wedge \mathrm{d} p\left(\gamma_{s}\right) .
\end{aligned}
$$

Our next step is to rewrite this expression in terms of residues at the marked puncture $P$. For this, we begin by changing coordinates on the Jacobian. Now the Baker-Akhiezer function $\psi(t, z)$ has $g$ zeroes $\gamma_{s}(t)$ on $\Gamma$ outside of the puncture $P$. The Riemann-Roch Theorem implies that these punctures coincide with the poles $\left(\gamma_{1}, \cdots, \gamma_{g}\right)$ when $t=0$. Otherwise, as $t$ varies, the dependence of the zeroes $\gamma_{s}(t)$ allows us to consider any set of $g$ times $t_{i_{1}}, \cdots, t_{i_{g}}$ for which the corresponding flows are independent, as a system of coordinates on the Jacobian $S^{g}(\Gamma)$. In general position, we can choose $t_{1}, \cdots, t_{g}$ as this system. The transformation from these coordinates to the system $\left(f\left(\gamma_{1}\right), \cdots, f\left(\gamma_{g}\right)\right)$ defined by an Abelian integral $f$ on $\Gamma$ is described by the following formula

$$
\partial_{t_{i}} f(\gamma(t))=\operatorname{Res}_{\gamma(t)} \frac{\partial_{t_{i}} \psi(t, z)}{\psi(t, z)} \mathrm{d} f .
$$

In the present case, we find

$$
\omega_{\mathcal{M}}=-\sum_{s=1}^{g} \operatorname{Res}_{\gamma_{s}}\left[\left.\frac{\left\langle\psi^{+} \delta L \psi\right\rangle}{\left\langle\psi^{+} \psi\right\rangle} \wedge \frac{\delta_{t} \psi}{\psi}\right|_{t=0}\right] \mathrm{d} p
$$

where

$$
\frac{\delta_{t} \psi}{\psi}=\sum_{j=1}^{g} \frac{\partial_{j} \psi(t, P)}{\psi(t, P)} \mathrm{d} t_{j}
$$

The variation $\delta_{t}$ can be viewed as the restriction of the full variation $\delta$ to a fiber of $\mathcal{N}^{g}$. In view of the properties of the dual Baker-Akhiezer function, the differential $\mathrm{d} \Omega$ of (18) can be recognized as [9]

$$
\mathrm{d} \Omega=\frac{\mathrm{d} p}{\left\langle\psi^{+} \psi\right\rangle} .
$$

Therefore, the differential on the right-hand side of (35) is a meromorphic differential with poles at $P$ and the points $\gamma_{s}=\gamma_{s}(0)$ only. Thus 
the sum of the residues at $\gamma_{s}$ is just the opposite of the residue at $P$

$$
\omega_{\mathcal{M}}=\operatorname{Res} P\left[\left.\frac{\left\langle\psi^{+} \delta L \psi\right\rangle}{\left\langle\psi^{+} \psi\right\rangle} \wedge \frac{\delta_{t} \psi}{\psi}\right|_{t=0}\right] \mathrm{d} p .
$$

Let $\delta J_{s}(t)$ be the coefficients of the expansion

$$
\frac{\psi^{+}(\delta L \psi)}{<\psi^{+} \psi>} \mathrm{d} p=-\sum_{s=1}^{\infty} \delta J_{s}(t) z^{-n-1+s} \mathrm{~d} z .
$$

The equation (31) implies that

$$
<\delta J_{s}(t)>_{x}=\delta H_{s}=0, s=1, \cdots, n-1,
$$

since the mean values $H_{s}$ are fixed along the leaf $\mathcal{M}$ (recall $<\cdot>_{x}$ denotes averages with respect to $x$, keeping $t_{n}=y$ fixed). In particular,

$$
\omega_{\mathcal{M}}=-\sum_{j=1}^{g}<\delta J_{j+n}(t)>\wedge \mathrm{d} t_{j} .
$$

Next, the differential

$$
\frac{\partial_{j} \psi^{+}(t, P)(\delta L \psi(t, P))}{<\psi^{+} \psi>} \mathrm{d} p
$$

is holomorphic on $\Gamma$ except at $P$. Therefore, its residue at this point is equal to zero and we obtain

$$
\delta J_{j+n}(t)=\sum_{s=1}^{n-1} \delta J_{s}(t) h_{n-s, i}^{+}(t)
$$

with $h_{s, j}^{+}(t)$ the coefficients of the expansion

$$
\frac{\partial_{j} \psi^{+}(t, P)}{\psi^{+}(t, P)}=-z^{-j}+\sum_{s=1}^{\infty} h_{s, j}^{+}(t) z^{s} .
$$

Therefore, (38) and (39) imply that

$$
\omega_{\mathcal{M}}=-\operatorname{Res}_{P} \frac{\left\langle\delta_{t} \psi^{+} \wedge \delta L \psi\right\rangle}{\left\langle\psi^{+} \psi\right\rangle} \mathrm{d} p
$$

We would like to replace the partial variation $\delta_{t}$ along the fiber in the preceding formula by the full variation $\delta$. To this effect, we show now 
that the residue of the contribution of the orthogonal variation $\delta^{E}$, i.e., the variation with fixed $E\left(\gamma_{s}\right)$, vanishes:

$$
\operatorname{Res}_{P} \frac{<\delta^{E} \psi^{+} \wedge \delta L \psi>}{\left\langle\psi^{+} \psi>\right.} \mathrm{d} p=0
$$

Indeed, the left-hand side can be rewritten as

$$
\delta^{E}\left(\operatorname{Res}_{P} \frac{<\log \psi^{+}\left(\psi^{+} \delta L \psi\right)>}{<\psi^{+} \psi>} \mathrm{d} p\right)=\delta^{E}\left(\sum_{s} 2 \pi i \int_{\gamma_{s}^{+}}^{\gamma_{s}^{+}(t)} \delta^{E} p \mathrm{~d} E\right)=0
$$

with the second equality a consequence of the main property of differentials, $\left(\delta^{E}\right)^{2}=0$. This establishes the first identity stated in Theorem 2 .

Next, it follows from (40) that

$$
\begin{aligned}
\delta \log \psi^{+} & =\delta\left(c\left(t_{2}, t_{3}, \ldots ; k\right)+\int_{x_{0}}^{x} \partial_{x} \ln \psi^{+}\right) \\
& =\delta \sum_{s=1}^{\infty}\left(c_{s}\left(t_{2}, t_{3}, \ldots, k\right)+\int_{x_{0}}^{x} h_{s}^{+} \mathrm{d} x\right) .
\end{aligned}
$$

Since $\left.<\delta J_{s}\right\rangle_{x}=0$, the above integration constants are immaterial, and we obtain

$$
\omega_{\mathcal{M}}=-\sum_{s=1}^{n-1}<\delta J_{s} \wedge \int_{x_{0}}^{x} \delta^{*} h_{n-s}^{+} \mathrm{d} x>
$$

From its definition (37), $\delta J_{s}$ does not contain variations of derivatives of $u_{i}$. Therefore, for $s=1, \ldots, n-1$,

$$
\delta J_{s}=-n \frac{\delta h_{s}}{\delta u} \delta u
$$

Substituting in the preceding equation gives the second identity in Theorem 2 .

3) The symplectic form for the Korteweg-de Vries Equation

The KdV equation corresponds to $n=2$. The relevant differential operator $L$ is then the second order differential operator

$$
L=\partial_{x}^{2}+u(x, y)
$$


and its coefficients are quasi-periodic functions $u(x, y)$ of two variables $\left(y=t_{2}\right)$. In this case the formula (29) becomes

$$
\omega_{\mathcal{M}}=-\frac{1}{2}<\delta u \wedge \int_{x_{0}}^{x} \delta u \mathrm{~d} x>
$$

and $\omega_{\mathcal{M}}$ is well-defined on a space of functions with fixed mean value in $x$ :

$$
<u>_{x}=H_{1} \longrightarrow<\delta u>_{x}=0 .
$$

The symplectic form (41) reduces to the Gardner-Faddeev-Zakharov symplectic form when $u(x, y)=u(x)$ is a function of one variable only. In this case, we get a reduction of the KP equation to the KdV equation

$$
u_{t}=\frac{3}{2} u u_{x}+\frac{1}{4} u_{x x x} .
$$

Indeed, for $n=2$, the differentials $\mathrm{d} p$ and $\mathrm{d} E$ at the puncture $P$ have the form

$$
\mathrm{d} p=\mathrm{d}\left(z^{-1}+O(z)\right), \quad \mathrm{d} E=\mathrm{d}\left(z^{-2}+O(z)\right) .
$$

Consider the leaf of the foliation defined by these differentials which corresponds to $\mathrm{d} E$ with all zero periods

$$
\oint_{C} \mathrm{~d} E=0
$$

for an arbitrary cycle $C$. In this case, the Abelian integral $E(P)$ is a single-valued function, with only a pole of second order at $P$. The corresponding curve is a hyperelliptic curve given by an equation of the form $y^{2}=\prod_{i=1}^{2 g+1}\left(E-E_{i}\right)$, and $P$ is the point at infinity $E=\infty$. For finite-gap solutions of the KdV equation, the periods

$$
a_{i}=\oint_{A_{i}} p \mathrm{~d} E
$$

are canonically conjugated (with respect to the Gardner-Faddeev-Zakharov symplectic structure) to the angle variables (see [14] and references therein). Our result is a generalization of this statement to the KP theory. Note that the leaves of the foliation corresponding to non-zero values of the periods of $\mathrm{d} E$ define a deformation of the space of hyperelliptic curves in the moduli space of arbitrary curves with one puncture. 


\section{4) The symplectic form for the Boussinesq Equation}

The Boussinesq equation corresponds to the case $n=3$, in which the operator $L$ is the third order differential operator

$$
L=\partial_{x}^{3}+u \partial_{x}+v .
$$

The fundamental formula (29) becomes

$$
\omega_{\mathcal{M}}=-\frac{1}{3}\left(\delta u \wedge \int_{x_{0}}^{x} \delta v \mathrm{~d} x+\delta v \wedge \int_{x_{0}}^{x} \delta u \mathrm{~d} x\right),
$$

which is a symplectic form on a space of two quasi-periodic functions $u=u(x, y), v=v(x, y)$ satisfying the constraints

$$
<u\rangle_{x}=\text { const },\langle v\rangle_{x}=\text { const. }
$$

The corresponding Poisson brackets have the form

$$
\{F, G\}=\frac{2}{3}\left(\frac{\delta F}{\delta u} \partial_{x} \frac{\delta G}{\delta v}+\frac{\delta G}{\delta u} \partial_{x} \frac{\delta F}{\delta v}\right) .
$$

The leaf of the foliation defined by the differentials $\mathrm{d} p$ and $\mathrm{d} E=\mathrm{d} \Omega_{3}$ with the periods of $d E$ all zero, corresponds to a reduction of the KP equation to the Boussinesq equation:

$$
u_{t}=2 v_{x}-u_{x x}, \quad v_{t}=v_{x x}-\frac{2}{3} u_{x x x}-\frac{2}{3} u u_{x} .
$$

Note that the usual form of the Boussinesq equation

$$
u_{t t}+\left(\frac{4}{3} u u_{x}+\frac{1}{3} u_{x x x}\right)_{x}=0
$$

as an equation on one unknown function $u$, is the result of eliminating $v$ from the system (43).

The system (43) is a Hamiltonian system with the bracket (42) and the following Hamiltonian

$$
H=\frac{3}{2}<v^{2}+u v_{x}+\frac{1}{3} u_{x}^{2}-\frac{1}{9} u^{3}>.
$$

In terms of $H$ the equations for $u$ and $v$ assume the remarkably simple form

$$
u_{t}=\frac{2}{3} \partial_{x} \frac{\delta H}{\delta v}, \quad v_{t}=\frac{2}{3} \partial_{x} \frac{\delta H}{\delta u} .
$$

We observe that the present Poisson bracket seems to differ from the well-known Gelfand-Dickey bracket [15] as well as from the generalized Gardner-Faddeev-Zakharov bracket. It would be interesting to understand better the relation between all of them. 


\section{Other integrable models}

It is straightforward to extend the preceding formalism to more general situations, including matrix cases, higher symplectic forms, and Calogero-Moser models. In this section, we discuss a few examples.

\section{The 2D Toda Lattice}

The 2D Toda lattice is the system of equations for the unknown functions $\varphi_{n}=\varphi_{n}\left(t_{+}, t_{-}\right)$:

$$
\frac{\partial^{2}}{\partial t_{+} \partial t_{-}} \varphi_{n}=e^{\varphi_{n}-\varphi_{n-1}}-e^{\varphi_{n+1}-\varphi_{n}}
$$

It is equivalent to the compatibility conditions for the following auxiliary linear problems

$$
\partial_{+} \psi_{n}=\psi_{n+1}+v_{n} \psi_{n}, v_{n}=\partial_{+} \varphi_{n}, \partial_{-} \psi_{n}=c_{n} \psi_{n-1}, c_{n}=e^{\varphi_{n}-\varphi_{n-1}} .
$$

A construction of algebraic-geometric solutions to the hierarchy of this system was proposed in [16].

Let $\Gamma$ be a smooth genus $g$ algebraic curve with fixed local coordinates $z_{ \pm}$in the neighborhoods of two punctures $P_{ \pm}$. Let $t=\left(t_{m, \pm}\right)_{m=1}^{\infty}$ be the set of time parameters. For any set of $g$ points $\gamma_{s}$ in general

position, the Baker-Akhiezer function is the unique function $\psi_{n}$ which is meromorphic on $\Gamma$ outside the punctures, has at most simple poles at the points $\gamma_{s}$, and admits the following singularities near $P_{ \pm}$:

$$
\psi_{n}\left(t, z_{ \pm}\right)=z_{ \pm}^{ - \pm n}\left(\sum_{s=0}^{\infty} \xi_{s}^{ \pm}(n, t) z_{ \pm}^{s}\right) \exp \left(\sum_{m=1}^{\infty} t_{m, \pm} z_{ \pm}^{-m}\right), \xi_{0}^{+} \equiv 1 .
$$

The times $t_{ \pm}$appearing in the Toda lattice correspond to $t_{1, \pm}$ in the Baker-Akhiezer function. We obtain a solution of the Toda lattice by setting

$$
\varphi_{n}=\log \xi_{0}^{-}(n, t) .
$$

The integer $n$ acts as a discrete space variable. It couples to the quasimomentum $p$, which is the Abelian integral characterized by the fact that $\mathrm{d} p$ has simple poles at the punctures $P_{ \pm}$with residues $\mp 1$ respectively, and is real-normalized. The differential which couples to the variable $t_{+}$is the diffferential $d \Omega_{+}$which has a pole at $P_{+}$of the form

$$
\mathrm{d} \Omega_{+}=\mathrm{d}\left(z_{+}^{-1}+O\left(z_{+}\right)\right) .
$$


Theorem 3. The symplectic form $\omega_{\mathcal{M}}$ corresponding to the differentials $\mathrm{d} p$ and $\mathrm{d} E=\mathrm{d} \Omega_{+}$is equal to

$$
\omega_{\mathcal{M}}=-\sum_{\alpha= \pm} \operatorname{Res}_{P_{\alpha}} \frac{<\delta \psi_{n}^{+} \wedge \delta L \psi_{n}>}{<\psi_{n}^{+} \psi_{n}>} \mathrm{d} p=<\delta \varphi_{n} \wedge \delta v_{n}>
$$

where $\varphi_{n}$ is the corresponding algebraic-geometric solution to the $2 \mathrm{D}$ Toda lattice, and $v_{n}=\partial_{+} \varphi_{n}$.

\section{2) Matrix Equations}

We show now that the algebraic-geometric symplectic form $\omega_{\mathcal{M}}$ generated by two real-normalized differentials $\mathrm{d} p$ and $\mathrm{d} E$ having poles of orders 2 and $n$ respectively at $N$ punctures $P_{\alpha}$, is a restriction of a symplectic form defined on a space of linear operators with matrix $(N \times N)$ coefficients.

The algebraic-geometric solutions to equations which have the zerocurvature representation

$$
\left[\partial_{y}-L, \partial_{t}-A\right]=0 \leftrightarrow L_{t}-A_{y}+[L, A]=0
$$

with

$$
L=\sum_{i=1}^{n} u_{i}(x, y, t) \partial_{x}^{i}, A=\sum_{i=1}^{m} v_{i}(x, y, t) \partial_{x}^{i}
$$

linear operators with matrix $(N \times N)$ coefficients, was constructed in [12] (an updated version is in [9]). The construction is based on the concept of vector Baker-Akhiezer function $\psi(x, y, t, z)$.

We fix a set of constants $a_{\beta}$ and a set of polynomials in $z^{-1}$

$$
q_{\beta}\left(z^{-1}\right)=\sum_{j=1}^{m_{\beta}} q_{j, \beta} z^{-j}
$$

Then for any smooth genus $g$ algebraic curve $\Gamma$ with fixed local coordinates $z_{\alpha}$ in the neighborhood of the punctures $P_{\alpha}$, and for any set of $g+N-1$ points $\gamma_{s} \in \Gamma$ in general position, $\psi_{\alpha}$ is the unique function which is meromorphic outside the punctures, has at most simple poles at the points $\gamma_{s}$, and has the following form in the neighborhood of the puncture $P_{\beta}$

$$
\psi_{\alpha}(x, y, t ; z)=\left(\delta_{\alpha, \beta}+\sum_{s=1}^{\infty} \xi_{s}^{\alpha, \beta}(x, y, t) z^{s}\right) e^{z^{-1} x+a_{\beta} z^{-n} y+q_{\beta}\left(z^{-1}\right) t},
$$

$$
z=z_{\beta} \text {. }
$$


The vector Baker-Akhiezer function $\psi(x, y, t, z)$ is defined by $\left(\psi_{\alpha}(x, y, t, z)\right)_{\alpha=1}^{N}$. There exists then unique operators $L$ and $A$ of the form (48) such that

$$
\left(\partial_{y}-L\right) \psi(x, y, t, P)=0,\left(\partial_{t}-A\right) \psi(x, y, t, P)=0 .
$$

The coefficients of the operators can be derived from the following system of linear equations

$$
\begin{aligned}
\sum_{i=0}^{n} u_{i} \sum_{l=0}^{i} C_{i}^{l}\left(\partial_{x}^{i-l} \xi_{s+l}\right)=\xi_{s+n} A, A^{\alpha, \beta} & =a_{\alpha} \delta^{\alpha, \beta}, \\
s & =-n, \ldots,-1,0, \\
\sum_{i=0}^{m} v_{i} \sum_{l=0}^{i} C_{i}^{l}\left(\partial_{x}^{i-l} \xi_{s+l}\right)=\sum_{j=1}^{m} \xi_{s+j} \hat{q}_{j}, \hat{q}_{j}^{\alpha, \beta} & =q_{j, \alpha} \delta^{\alpha, \beta}, \\
s & =-m, \cdots,-1,0,
\end{aligned}
$$

In particular, we have

$$
u_{n}=A, u_{n-1}=\left[\xi_{1}, A\right], u_{n-2}=\left[\xi_{2}, A\right]-n \partial_{x} \xi_{1}, \ldots
$$

The defining condition (50) implies that the operators $L$ and $A$ satisfy the compatibility conditions (47), and hence are solutions of the corresponding non-linear equations.

We also require the concept of the dual vector Baker-Akhiezer function $\psi^{+}(x, y, t, P)$, which should be considered as row-vector, whose components $\psi_{\alpha}^{+}$are characterized by the following analytical properties. Let $\mathrm{d} \Omega$ be a unique differential with poles of the form

$$
\mathrm{d} \Omega=\mathrm{d}\left(z_{\alpha}^{-1}+O\left(z_{\alpha}\right)\right)
$$

at the punctures $P_{\alpha}$, and with zeros at the marked points $\gamma_{s}$. In addition to $\gamma_{s}$, it has $g+N-1$ other zeros which we denote $\gamma_{s}^{+}$. Then $\psi_{\alpha}^{+}$is the unique function which is meromorphic outside the punctures, has at most simple poles at the points $\gamma_{s}^{+}$, and has the form

$$
\begin{array}{r}
\psi_{\alpha}^{+}(x, y, t, z)=\left(\delta_{\alpha, \beta}+\sum_{s=1}^{\infty} \xi_{s}^{\alpha, \beta ;+}(x, y, t) z^{s}\right) e^{-z^{-1} x-a_{\beta} z^{-n} y-q_{\beta}\left(z^{-1}\right) t}, \\
z=z_{\beta},
\end{array}
$$


in the neighborhood of the puncture $P_{\beta}$.

Theorem 4. The restriction of the form

$$
\omega_{\mathcal{M}}=\delta\left(\sum_{s=1}^{g+N-1} p\left(\gamma_{s}\right) \mathrm{d} E\left(\gamma_{s}\right)\right)
$$

to the fibration $\mathcal{N}^{g+N-1}$ over the leaf $\mathcal{M}$ in $\mathcal{M}_{g}(n, 2)$ given by realnormalized meromorphic differentials $\mathrm{d} p$ and $\mathrm{d} E$ with poles at the punctures of the form

$$
\mathrm{d} p=\mathrm{d}\left(z^{-1}+O(z)\right), \quad \mathrm{d} E=\mathrm{d}\left(a_{\alpha} z^{-n}+O(z)\right)
$$

is equal to

$$
\omega_{\mathcal{M}}=-\sum_{\alpha=1}^{N} \operatorname{Res}_{P_{\alpha}} \frac{\left\langle\delta \psi^{+} \wedge \delta L \psi\right\rangle}{\left\langle\psi^{+} \psi\right\rangle} \mathrm{d} p .
$$

The proof of the theorem is essentially identical to that of the scalar case. We observe that, although the proof relies on the vector BakerAkhiezer function as a function which depends on a infinite number of times $t_{\alpha, i}$, the statement of the theorem itself uses only three marked times $(x, y, t)$.

As a concrete example, we discuss the $N$-wave equation. Consider the case $n=1$. In this case the operator $L$ has the form

$$
L=A \partial_{x}+u(x, y),
$$

where $A$ is the $N \times N$ matrix

$$
A^{\alpha, \beta}=a_{\alpha} \delta_{\alpha, \beta},
$$

and $u(x, y)$ is an $N \times N$ matrix with zero diagonal entries $u^{\alpha, \alpha}=0$. As shown in [12] and references therein, for $m=1$, the leaves corresponding to zero periods of $\mathrm{d} E$ give solutions to the so called $N$-wave equation.

The equations (51)-(52) imply that

$$
u^{\alpha, \beta}=\left(a_{\beta}-a_{\alpha}\right) \xi_{1}^{\alpha, \beta} .
$$

From the definition of the dual Baker-Akhiezer function it follows that

$$
\xi_{1}^{\alpha, \beta}+\xi_{1}^{\beta, \alpha ;+}=0 .
$$


Thus the right-hand side of the formula (55) defines the following symplectic form $\omega_{\mathcal{M}}$ on the space of matrix-functions $u$ with zero diagonal elements:

$$
\omega_{\mathcal{M}}=\sum_{\alpha \neq \beta} \frac{1}{a_{\alpha}-a_{\beta}} \delta u^{\beta, \alpha} \wedge \delta u^{\alpha, \beta}
$$

\section{3) Higher symplectic forms}

So far we have considered only the cases where the differential $\mathrm{d} Q$ has poles of order 2 at the punctures $P_{\alpha}$, or simple poles with resides $\mp 1$ at the punctures $P_{\alpha}^{ \pm}$. In these cases d $Q$ may be identified with the differential of the quasi-momentum coupled with the marked variable $x$ in the case of differential operators, or the discrete variable $n$ in the case of difference operators.

We consider now the general case. Let $\mathrm{d} E$ and $\mathrm{d} Q$ be real-normalized differentials having at the puncture $P_{\alpha}$ the form

$$
\mathrm{d} E=a_{\alpha} \mathrm{d}\left(z^{-n}+O(z)\right), \quad \mathrm{d} Q=\mathrm{d}\left(q_{\alpha}\left(z^{-1}\right)+O(z)\right) .
$$

If the polynomials $q_{\alpha}\left(z^{-1}\right)$ are the same as in (49), the differentials $\mathrm{d} E$ and $\mathrm{d} Q$ reduce to the differentials of the quasi-momenta for the corresponding Baker-Akhiezer function with respect to the variables $y$ and $t$.

Theorem 5. The restriction of the form

$$
\omega_{\mathcal{M}}=\delta\left(\sum_{s=1}^{g+N-1} Q\left(\gamma_{s}\right) \mathrm{d} E\left(\gamma_{s}\right)\right)
$$

to a leaf of the corresponding foliation is equal to

$$
\omega_{\mathcal{M}}=\sum_{\alpha=1}^{N} \operatorname{Res}_{P_{\alpha}} \frac{\left\langle\delta \psi^{+} \wedge\left(L^{(1)} \delta A-A^{(1)} \delta L\right) \psi>\right.}{\left\langle\psi^{+} \psi\right\rangle} \mathrm{d} p,
$$

where $L^{(1)}$ and $A^{(1)}$ are the first descendants of the operators $L$ and $A$ given in (48):

$$
L^{(1)}=\sum_{i=1}^{n} i u_{i}(x, y, t) \partial_{x}^{i-1}, \quad A^{(1)}=\sum_{i=1}^{m} i v_{i}(x, y, t) \partial_{x}^{i-1} .
$$


The proof of this theorem follows the same lines as the proof of Theorem 2 , with the identity (31) replaced by the following generalization, whose proof was also given in [9] (the additional terms in [9] cancel on the leaves we are considering)

$$
\delta Q(E)=\frac{<\psi^{+}\left(L^{(1)} \delta A-A^{(1)} \delta L\right) \psi>}{\left\langle\psi^{+} \psi>\right.} \frac{\mathrm{d} Q}{\mathrm{~d} E}
$$

As an example, consider the case where the number of punctures $N$ is $1, n$ is 3 , and $m$ is 2 . The corresponding operators $L$ and $A$ are

$$
L=\partial_{x}^{3}+u \partial_{x}+v, \quad A=\partial_{x}^{2}+\frac{2}{3} u .
$$

The operators $L^{(1)}$ and $A^{(1)}$ are then given by

$$
L^{(1)}=3 \partial_{x}^{2}+u, A=2 \partial_{x}^{1},
$$

and the identity (58) becomes

$$
\begin{aligned}
\omega_{\mathcal{M}}=< & \frac{3}{4} u \delta u \wedge \int_{x_{0}}^{x} \delta u \mathrm{~d} x+2 \delta v \wedge \int_{x_{0}}^{x} \delta v \mathrm{~d} x \\
& +2 \delta u \wedge \delta v-\frac{3}{2} \delta u_{x} \wedge \delta u>.
\end{aligned}
$$

The right-hand side is well-defined on a space of two quasi-periodic functions $u(x, y)$ and $v(x, y)$ satisfying the constraints

$$
<u>_{x}=\text { const },<v>_{x}=\text { const },<u^{2}>_{x}=\text { const } .
$$

\section{4) The elliptic Calogero-Moser System}

Until now we have only discussed systems where the Lax pair $L, A$ does not contain a spectral parameter. However, there is strong evidence that the present approach is quite general, and can extend to this case as well. A good example is the elliptic Calogero-Moser system, which we discuss next.

The elliptic Calogero-Moser system [17] is a system of $N$ identical particles on a line interacting with each other via the potential $V(x)=$ $\wp(x)$, where $\wp(x)=\wp\left(x \mid \omega, \omega^{\prime}\right)$ is the Weierstrass elliptic function with 
periods $2 \omega, 2 \omega^{\prime}$. The complete solution of the elliptic Calogero-Moser system was constructed by algebraic-geometric methods in [8]. There it was found that the equations of motion

$$
\ddot{x}_{i}=4 \sum_{j \neq i} \wp^{\prime}\left(x_{i}-x_{j}\right), \quad \wp(x)=\frac{d \wp(x)}{d x}
$$

have a Lax representation depending on a spectral parameter $z$. The Lax operator $L$ has the form:

$$
L_{i j}(t, z)=p_{i} \delta_{i j}+2\left(1-\delta_{i j}\right) \Phi\left(x_{i}-x_{j}, z\right), p_{i}=\dot{x}_{i},
$$

with $\Phi(x, z)$ given by

$$
\Phi(x, z)=\frac{\sigma(z-x)}{\sigma(z) \sigma(x)} e^{\zeta(z) x} .
$$

The characteristic polynomial

$$
R(k, z)=\operatorname{det}(2 k+L(t, z))
$$

is time-independent, and defines a time-independent spectral curve $\Gamma$ by the equation

$$
R(k, z) \equiv \sum_{i=0}^{N} r_{i}(z) k^{i}=0,
$$

where the $r_{i}(z)$ are elliptic functions of $z$. The Jacobians $J(\Gamma)$ of the spectral curves $\gamma$ are levels of the involutive integrals of the system. In particular, we obtain angle variables $\varphi_{i}$ by choosing $2 \pi$-periodic coordinates on them. It should be mentioned that although the exact solution to the Calogero-Moser system in terms of $\Theta$-functions was found in [16], the action-variables $a_{i}$ canonically conjugated to the angle-variables $\varphi_{i}$ were found only relatively recently in [18]. There it was shown that the Calogero-Moser sytem can be obtained through a Hamiltonian reduction from the Hitchin system, and as a result, the action-variables $a_{i}$ are the periods of the differential $k d z$ along $A$-cycles of the spectral curve $\Gamma$.

We show now that this statement can be obtained directly from our approach. Let $C(P)=\left(c_{1}, \ldots, c_{N}\right)$ and $C^{+}(P)=\left(c_{1}^{+}, \ldots, c_{N}^{+}\right)$be solutions of the linear equations

$$
(2 k+L(z)) C=0, C^{+}(2 k+L(z))=0,
$$


where $C$ is considered as a column-vector, $C^{+}$as a row-vector, and $P=(k, z)$ is a point of the spectral curve $\Gamma$. If we normalize the eigenvectors $C$ and $C^{+}$by the conditions

$$
\sum_{i} c_{i} \Phi\left(-x_{i}, z\right)=1, \sum_{i=1} c_{i}^{+} \Phi\left(x_{i}, z\right)=1,
$$

then it follows from [8] that $C(P)$ and $C^{+}(P)$ are meromorphic functions on $\Gamma$ outside the points $P_{\alpha}$ on $\gamma$ corresponding to $z=0$, and have $N$ poles. We denote these poles by $\gamma_{1}, \cdots, \gamma_{N}$ and $\gamma_{1}^{+}, \ldots, \gamma_{N}^{+}$, respectively. Near the points $P_{\alpha}$, the components of these vectors have the form

$$
c_{i}(P)=\left(c_{i}^{\alpha}+O(z)\right) e^{x_{i} z^{-1}}, \quad c_{i}^{+}(P)=\left(c_{i}^{\alpha,+}+O(z)\right) e^{-x_{i} z^{-1}}
$$

and

$$
\begin{aligned}
c_{i}^{1} & =1 ; \sum_{i} c_{i}^{\alpha}=0 \text { for } \alpha>1, \\
c_{i}^{1,+} & =1 ; \sum_{i} c_{i}^{\alpha,+}=0 \text { for } \alpha>1 .
\end{aligned}
$$

The formalism in this paper applies exactly as before to produce the following formula for the symplectic form for the Calogero-Moser system

$$
2 \delta\left(\sum_{s=1}^{N} k\left(\gamma_{s}\right) d z\right)=\sum_{\alpha=1}^{N} \operatorname{Res}_{P_{\alpha}} \frac{\left\langle\delta C^{+} \wedge \delta L C\right\rangle}{\left\langle C^{+} C\right\rangle} d z,
$$

with $\left\langle f^{+} g\right\rangle$ the usual pairing between vectors and co-vectors. Substituting in the expansion (68), (69), we obtain at once

$$
\delta\left(\sum_{s=1}^{N} k\left(\gamma_{s}\right) d z\right)=\frac{1}{2} \sum_{i}^{N} \delta p_{i} \wedge \delta x_{i} .
$$

\section{V. $N=2$ supersymmetric $\mathbf{S U}\left(N_{c}\right)$ gauge theories}

We turn now to a discussion of the universal configuration space $\mathcal{M}_{g}(n, m)$ and of the symplectic forms $\omega_{\mathcal{M}}$ in the context of $\mathrm{N}=2$ supersymmetric Yang-Mills theories with gauge group $\mathrm{SU}\left(N_{c}\right)$.

We consider gauge theories with $N_{c}$ colors and $N_{f}$ flavors. The field content is an $\mathrm{N}=2$ chiral multiplet and $N_{f}$ hypermultiplets of bare 
masses $m_{i}$. The $\mathrm{N}=2$ chiral multiplet contains a complex scalar field $\phi$ in the adjoint representation of $\mathrm{SU}\left(N_{c}\right)$. Classically, the flat directions in the potential correspond to $\phi$ lying in the Cartan subalgebra. Thus the theory admits a $\left(N_{c}-1\right)$-dimensional space of vacua, which can be parametrized by the order parameters

$$
u_{k} \equiv \operatorname{Tr}<\phi^{k}>, \quad k=2, \cdots, N_{c} .
$$

It is often more convenient to work with the parameters $s_{i}, i=0, \cdots, N_{c}$, defined recursively by $s_{0}=0, s_{1}=u_{1}=0$, and

$$
k s_{k}+\sum_{i=1}^{k} s_{k-i} u_{i}=0 .
$$

In the weak coupling limit, the $s_{k}$ 's correspond to $k$ th symmetric polynomials in $\phi$, but they do not of course admit in general such a simple interpretation. In the $\mathrm{N}=1$ formalism, the effective Lagrangian is of the form

$$
\mathcal{L}=\operatorname{Im} \frac{1}{4 \pi}\left[\int d^{4} \theta \partial_{i} \mathcal{F}(A) \bar{A}^{i}+\frac{1}{2} \int d^{2} \theta \partial_{i} \partial_{j} \mathcal{F}(A) W^{i} W^{j}\right],
$$

where the $A_{i}$ 's are $\mathrm{N}=1$ chiral superfields whose scalar components correspond to the eigenvalues of $\phi$. The holomorphic pre-potential $\mathcal{F}$ as well as the spectrum of BPS states can all be determined by finding a fibration of spectral curves as well as of Seiberg-Witten forms $d \lambda$ over the moduli space of vacua, and setting

$$
\begin{aligned}
& a \equiv \oint_{A} \mathrm{~d} \lambda, \quad a_{D} \equiv \oint_{B} \mathrm{~d} \lambda, \quad \frac{\partial \mathcal{F}}{\partial a_{i}}=a_{D, i}, \\
& M_{B P S}^{2}=2|Z|^{2}, \quad Z=\sum_{i=1}^{N_{c}}\left[n_{e}^{i} a_{i}+n_{m}^{i} a_{D, i}\right]+\sum_{i=1}^{N_{f}} S_{i} m_{i} .
\end{aligned}
$$

Here the $S_{i}$ 's are U(1) charges corresponding to global symmetries, and $n_{e}^{i}$ and $n_{m}^{i}$ are respectively electric and magnetic charges. Some key requirements for $\Gamma$ and $\mathrm{d} \lambda$ are e.g. consistency with the weak coupling evaluation of the monodromies of the effective Lagrangian as the gauge bosons become massless, with instanton contributions, with the classical limit, and with an infinite mass limit reducing the number of flavors [1], $[3]$.

We shall construct the desired fibration of spectral curves and vector bundle simply by identifying the moduli space of gauge vacua with an 
appropriate leaf $\mathcal{M}$ in our universal configuration space $\mathcal{M}_{g}(n, m)$, for a suitable choice of $n, m$ and $g$. Thus set, in the notation of Section II,

$$
\begin{aligned}
g & =N_{c}-1,\left(P_{\alpha}\right)=\left(P_{+}, P_{-} ; P_{1}, \cdots P_{N_{f}}\right), \\
m & =\left(m_{+}, m_{-} ; m_{1}, \cdots, m_{N_{f}}\right)=(1,1 ; 0, \cdots, 0), \\
n & =\left(n_{+}, n_{-} ; n_{1}, \cdots, n_{N_{f}}\right)=(0,0 ; 0, \cdots, 0) .
\end{aligned}
$$

In the resulting configuration space $\mathcal{M}_{g}(n, m)$, consider the leaf $\mathcal{M}_{N_{c}, N_{f}}$ characterized by the following conditions on the singular parts of the Abelian integrals $E$ and $Q$

$$
\begin{aligned}
R_{\alpha}^{E} & =1 \text { for } 1 \leq \alpha \leq N_{f}, \quad R_{-}^{E}=N_{c}-N_{f}, \\
R_{\alpha}^{Q} & =0 \text { for } 1 \leq \alpha \leq N_{f}, \quad R_{-}^{Q}=0, \\
T_{\alpha, 0} & =-m_{\alpha}, \quad 1 \leq \alpha \leq N_{f}, \quad T_{+, 0}=0, \\
T_{+, 1} & =-N_{c} 2^{-1 / N_{c}}, \\
T_{-, 1} & =\left(N_{c}-N_{f}\right)\left(\frac{\Lambda}{2}\right)^{1 /\left(N_{c}-N_{f}\right)}, \\
T_{\alpha, 1} & =0,1 \leq \alpha \leq N_{f},
\end{aligned}
$$

and the following conditions on their periods

$$
\begin{gathered}
\oint_{A} \mathrm{~d} Q=\oint_{B} \mathrm{~d} Q=0 \\
\frac{1}{2 \pi i} \oint_{A} \mathrm{~d} E=m \in \mathbf{Z}^{N_{c}-1}, \quad \frac{1}{2 \pi i} \oint_{B} \mathrm{~d} E=n \in \mathbf{Z}^{N_{c}-1} .
\end{gathered}
$$

The conditions on $Q$ imply that $Q$ is actually a well-defined meromorphic function on $\Gamma$, with only simple poles at $P_{ \pm}$. The third condition in (76) means that $Q\left(P_{i}\right)=-m_{i}$. Now a point of the universal configuration space also requires a jet of coordinates at each puncture $P_{ \pm}$and $P_{i}$. In the present case, these jets are provided by the function $Q$, more precisely, by $Q^{-1}$ at $P_{ \pm}$, and by $Q+m_{i}$ at $P_{i}$.

The form $\mathrm{d} E$ is a meromorphic form with only simple poles at $P_{i}$ and $P_{ \pm}$. Its residues at $P_{i}$ are 1 , while its residues at $P_{+}$and $P_{-}$are respectively $-N_{c}$ and $N_{c}-N_{f}$. The form $\mathrm{d} \lambda=Q \mathrm{~d} E$ is a single-valued meromorphic 1-form on $\Gamma$. We identify it with the Seiberg-Witten form of the gauge theory. Since its residues at $P_{i}, 1 \leq i \leq N_{f}$, are $-m_{i}$, we recognize the parameters $m_{i}, 1 \leq i \leq N_{f}$, as the masses of the 
hypermultiplets in the gauge theory. The residues of $\mathrm{d} \lambda$ depend then linearly on the masses, as they should in order for the BPS spectrum (c.f. (74)) to be expressible in terms of the periods of $d \lambda$. We shall see shortly that the remaining parameter $\Lambda$ in (76), can be interpreted as essentially the dynamically generated scale of the gauge theory.

We pause briefly to discuss the holomorphicity of the derivatives along $\mathcal{M}_{N_{c}, N_{f}}$ of $Q \mathrm{~d} E$. As we have seen in Section II, the notion of derivatives requires a connection, which is in the case provided by the level sets of the Abelian integral $E$. It is with respect to this connection, $\nabla^{E}$, that the derivatives of $Q \mathrm{~d} E$ are holomorphic. However, the roles of $E$ and $Q$ are almost interchangeable, and we can consider $\nabla^{Q}(Q \mathrm{~d} E)$ as well. On functions $f$, the two connections are related by

$$
\nabla_{X}^{E} f=\nabla_{X}^{Q} f-\frac{\mathrm{d} f}{\mathrm{~d} E} \nabla_{X}^{Q} E
$$

With respect to the connection $\nabla^{Q}$, the derivatives of $Q \mathrm{~d} E$ will develop poles. However, interchanging the roles of $E$ and $Q$, we can see that $\nabla^{Q}(E \mathrm{~d} Q)$ is holomorphic. In fact, equation (79) implies that

$$
\nabla^{E}(Q \mathrm{~d} E)=\left(\nabla^{E} Q\right) \mathrm{d} E=\left(\nabla^{Q} Q-\frac{\mathrm{d} Q}{\mathrm{~d} E} \nabla^{Q} E\right) \mathrm{d} E=-\nabla^{Q}(E \mathrm{~d} Q)
$$

This shows that at the level of derivatives along $\mathcal{M}_{N_{c}, N_{f}}$, the two forms $Q \mathrm{~d} E$ and $E \mathrm{~d} Q$ are interchangeable. However, for the leaf $\mathcal{M}_{N_{c}, N_{f}}$ we are discussing, only $Q \mathrm{~d} E$ is well-defined.

We derive now the equation of the curve $\Gamma$. First, it follows from the existence of a meromorphic function $Q$ with exactly two simple poles that $\Gamma$ is a hyperelliptic Riemann surface. Next, the integrality conditions (78) imply that, although $E$ is a multiple-valued Abelian integral, the function

$$
w=\exp E
$$

is a well-defined meromorphic function on $\Gamma$, with only poles at $P_{ \pm}$, of order respectively $N_{c}$ and $N_{f}-N_{c}$. As a consequence, there exists polynomials $A(Q)$ and $B(Q)$ of degrees respectively $N_{c}$ and $N_{f}$ such that

$$
w+\frac{B(Q)}{w}=2 A(Q)
$$


At the (finite) zeroes $P_{i}$ of $w, Q\left(P_{i}\right)=-m_{i}$, and thus $B(Q)$ must be of the form

$$
B(Q)=\tilde{\Lambda} \prod_{i=1}^{N_{f}}\left(Q+m_{i}\right)
$$

for a suitable constant $\tilde{\Lambda}$. We shall show that this parameter $\tilde{\Lambda}$ is the same as the earlier $\Lambda$ parametrizing the leaf. For this, we consider the expansion of the Abelian integral $E$ near $P_{+}$in terms of $Q$. Recall that the coordinate $z$ appearing in the definition of $T_{+, k}$ is defined by $E=-N_{c} \log z$. Thus the fourth condition in (76) means that $Q=$ $2^{-1 / N_{c}} z^{-1}+O(1)$, and we have near $P_{+}$

$$
E=N_{c} \log Q+\log 2+O\left(Q^{-1}\right) .
$$

Similarly, near $P_{-}$, we have

$$
E=-\left(N_{c}-N_{f}\right) \log Q+\log 2+\log \frac{\Lambda}{4}+O\left(Q^{-1}\right) .
$$

This means in particular that the parameter $\Lambda$ of (76) can be interpreted as the regularized version of the following logarithmically divergent integral

$$
\log \frac{\Lambda}{4}=\left(\int_{P_{+}}^{P_{-}} \mathrm{d} E\right)+\left.\left(2 N_{c}-N_{f}\right) \log Q\right|_{Q \rightarrow \infty} .
$$

This is a confirmation of the interpretation of $\Lambda$ as a scale, since the classical limit $\Lambda \rightarrow 0$ does correspond to the two poles $P_{ \pm}$becoming infinitely separated, and to the curve $\Gamma$ degenerating to the classical curve. We can now determine $\tilde{\Lambda}$ by evaluating the right-hand side of (86), using the equation for $w$. We find

$$
\int_{P_{+}}^{P_{-}} \mathrm{d} E=-\left.\log \frac{A+\sqrt{A^{2}-B}}{A-\sqrt{A^{2}-B}}\right|_{Q=\infty}=-\left(2 N_{c}-N_{f}\right) \log Q+\log \frac{\tilde{\Lambda}}{4},
$$

so that $\Lambda$ agrees with $\tilde{\Lambda}$.

We can also write the form $\mathrm{d} \lambda=Q \mathrm{~d} E$ in terms of the polynomials $A(Q)$ and $B(Q)$. Setting $y=w-A(Q)$, we find

$$
\mathrm{d} \lambda=\frac{Q}{y}\left(A^{\prime}-\frac{1}{2} \frac{B^{\prime}}{B}(A-y)\right) \mathrm{d} Q .
$$


The poles $P_{1}, \cdots, P_{N_{f}}$ of this form $\mathrm{d} \lambda$ occur on only one sheet of the hyperelliptic surface $\Gamma$. The symmetric version with poles on both sheets adopted by Seiberg and Witten [2] (see also Hanany and $\mathrm{Oz}[3]$ ) can be obtained by symmetrizing

$$
\mathrm{d} \tilde{\lambda}=\frac{Q}{y}\left(A^{\prime}-\frac{1}{2} \frac{A B^{\prime}}{B}\right) \mathrm{d} Q .
$$

We note that this does not affect the physics, since the difference $\frac{Q B^{\prime}}{2 B} \mathrm{~d} Q$ is $y$-independent, and hence has zero periods.

As we can see from Theorem 1, a natural parametrization of the leaf $\mathcal{M}_{N_{c}, N_{f}}$ is in terms of the periods $a_{i}$ of $\mathrm{d} \lambda=Q \mathrm{~d} E$ along $A$-cycles. This is particularly attractive from the viewpoint of gauge theories, since the $a_{i}$ 's are the scalar components of the light superfields in the effective theory. The dual fields $a_{D, i}$ as well as the effective Lagrangian $\mathcal{F}$ are already determined by our formalism without having to parametrize $\mathcal{M}_{N_{c}, N_{f}}$ specifically in terms of the order parameters $u_{k}$ of the gauge theory. The same is true of all the information we have obtained so far on the equation of the curve $\Gamma$ itself.

To write $\Gamma$ in terms of the $u_{k}$ 's, we consider the expansion of $E$ near $P_{+}$, and set

$$
E=N_{c} \log Q+\log 2+\log \left(1+\sum_{i=1}^{\infty} s_{i} Q^{-i}\right)
$$

In view of the third constraint in $(76)$, the coefficient $s_{1}$ is 0 . The next $N_{c}-1$ coefficients $s_{2}, \cdots, s_{N_{c}}$ provide independent coordinates for the leaf $\mathcal{M}_{N_{c}, N_{f}}$. We identify them with the order parameters $s_{i}$ of the gauge theory, as defined by (73).

With this identification, it is easy to determine all the coefficients of $A(Q)$ in terms of the $s_{k}$ 's, since the Abelian integral $E$ is also given by the equation

$$
E=\log w=\log \left(A+\left(A^{2}-B\right)^{1 / 2}\right) .
$$

We shall work it out explicitly when $N_{f} \leq N_{c}+1$. Set

$$
A(Q)=\sum_{i=0}^{N_{c}} c_{i} Q^{N_{c}-i}
$$


In this case, we may rewrite on one hand the expansion (89) as

$$
E=\log 2+\log \left(Q^{N_{c}}+\sum_{i=2}^{N_{c}} s_{i} Q^{N_{c}-i}+O\left(Q^{-1}\right)\right),
$$

and on the other hand, the expansion (90) as

$$
E=\log 2+\log \left(A-\frac{1}{4} \frac{B}{A}+O\left(Q^{-1}\right)\right) .
$$

Comparing the two equations, we find that $A(Q)$ is monic, and that $c_{1}=s_{1}=0$. This implies in turn that $A^{-1}=Q^{-N_{c}}\left(1+O\left(Q^{-2}\right)\right)$, and we obtain for $N_{f} \leq N_{c}+1$

$$
Q^{N_{c}}+\sum_{i=2}^{N_{c}} s_{i} Q^{N_{c}-i}=A-\frac{1}{4} B Q^{-N_{c}}+O\left(Q^{-1}\right) .
$$

Altogether, setting

$$
y=w-A(Q), t_{k}(m)=\sum_{i_{1}<\cdots<i_{k}} m_{i_{1}} \cdots m_{i_{k}},
$$

and making the identification $\Lambda \rightarrow \Lambda_{N_{c}, N_{f}}^{2 N_{c}-N_{f}}$, where $\Lambda_{N_{c}, N_{f}}^{2 N_{c}-N_{f}}$ is the dynamically generated scale of the theory, we have shown

Theorem 6. Let $\mathcal{M}_{N_{c}, N_{f}}$ be the leaf of the universal configuration space $\mathcal{M}_{g}(n, m)$ defined by the level sets (75), , , (78), and define $\mathrm{d} \lambda$ as $\mathrm{d} \lambda=Q \mathrm{~d} E$. Then $\mathcal{M}_{N_{c}, N_{f}}$ can be parametrized by the A-periods $a_{i}$ 's of $\mathrm{d} \lambda$, or by the coefficients $s_{2}, \cdots, s_{N_{c}}$ of (73). The equation of the curves $\Gamma$ fibering over $\mathcal{M}_{N_{c}, N_{f}}$ is $y^{2}=A(Q)^{2}-B(Q)$, with $B(Q)$ given by (83). For $N_{f} \leq N_{c}+1$, the equation of the curve $\Gamma$ takes the form suggested by Hanany and $\mathrm{Oz}$

$$
\begin{aligned}
y^{2}= & \left(\sum_{i=0}^{N_{c}} s_{i} Q^{N_{c}-i}+\frac{\Lambda_{N_{c}, N_{f}}^{2 N_{c}-N_{f}}}{4} \sum_{k=0}^{N_{f}-N_{c}} t_{k}(m) Q^{N_{f}-N_{c}-k}\right)^{2} \\
& -\Lambda_{N_{c}, N_{f}}^{2 N_{c}-N_{f}} \prod_{i=1}^{N_{f}}\left(Q+m_{i}\right) .
\end{aligned}
$$

Finally, we note a possible interpretation, in the context of integrable models, for both the Abelian integrals $E$ and $Q$ appearing in the form 
$\mathrm{d} \lambda$ of supersymmetric gauge theories. For pure $\mathrm{SU}\left(N_{c}\right), Q$ corresponds to the eigenvalues of the operator $(L \psi)_{n}=\psi_{n+1}+v_{n} \psi_{n}+c_{n-1} \psi_{n-1}$ defining the periodic Toda chain, and $\exp E$ to the eigenvalues of the monodromy operator $(T \psi)_{n}=\psi_{n+N_{c}}$. In presence of hypermultiplets, the periodic boundary condition gets shifted to an aperiodic Toda chain, with the "monodromy" operator $T$ rather of the form $(T \psi)_{n}=\psi_{n+N_{c}}+$ $\sum_{\kappa=1}^{N_{f}} \lambda_{n, \kappa} \psi_{n+N_{c}-\kappa}$.

In [11], Gorsky et al. suggest other representations in terms of spin chains. It would be interesting to understand better the relation between these different constructions.

\section{Appendix}

In this Appendix, we provide a proof of Theorem 1.

Assume that the differentials of all the coordinates are linearly dependent at a point of our universal configuration space. Then there exists a one-parameter deformation of this point (i.e., a one-parameter deformation of the curve $\Gamma^{t}$ and of all the other data) with the derivative $\left.\partial_{t}\right|_{t=0}$ of all the data equal to zero. In particular

$$
\left.\partial_{t}(Q(E, t) \mathrm{d} E)\right|_{t=0}=0,
$$

since the left-hand side is a holomorphic differential on $\Gamma^{0}$ with zero periods and must be identically zero. Let $\gamma$ be a point of the zero divisor of $\mathrm{d} E$ on $\Gamma^{0}$. For simplicity, we consider first the case where this point is a simple zero. Then, locally, the equation $\mathrm{d} E(\gamma(t))=0, \gamma(t) \in \Gamma^{t}$ defines a deformation of $\gamma$. In a neighborhood of our point on the universal configuration space, the Abelian integral $Q$ is a holomorphic function in this neighborhood, and has an expansion of the form

$$
Q(E, t)=q_{0}(t)+\sum_{i=1}^{\infty} q_{i}(t)\left(E-E_{t}\right)^{i / 2},
$$

where $E_{t}$ is a critical value of Abelian integral $E$ :

$$
E_{t}=E\left(\gamma_{t}\right)
$$

and the $q_{i}$ 's are analytic functions of the variable $t$. Since

$$
\left.\mathrm{d} Q(E, t)=q_{1}(t) \frac{\mathrm{d} E}{\sqrt{E-E_{t}}}+q_{2} \mathrm{~d} E+O\left(E-E_{t}\right)^{1 / 2}\right) \mathrm{d} E
$$


and thus $q_{1}(0) \neq 0$ in view of the assumptions of Theorem 1 , the equality

$$
\left.\partial_{t} Q(E, t)\right|_{t=0}=\frac{\left.q_{1}(0) \partial_{t} E_{t}\right|_{t=0}}{\sqrt{\left(E-E_{t}\right)}}+O(1)
$$

and (A.2) imply

$$
\left.\partial_{t} E_{t}\right|_{t=0}=0 .
$$

Next, we show that (A.6) yields in turn that

$$
\left.\partial_{t} \omega_{i}(E, t)\right|_{t=0}=0,
$$

where

$$
\omega_{i}(E, t)=\int_{P_{1}^{t}}^{E} \mathrm{~d} \omega_{i}
$$

is the Abelian integral of the normalized holomorphic differential $\mathrm{d} \omega_{i}$. Indeed, the left-hand side of (A.7) is an Abelian integral with possibly poles at the zeros of $\mathrm{d} E$. Its expansion at these zeros similar to (A.2) leads to that it is holomorphic at these points due to (A.6). Thus the left-hand side of (A.7) is a holomorphic Abelian integral with zero $A$ periods, and must be identically 0 . Its $B$-periods must also be zero, and hence

$$
\left.\partial_{t} \tau_{i j}(t)\right|_{t=0}=0,
$$

where $\tau_{i j}(t)$ is the period matrix of $\Gamma^{t}$. In view of the infinitesimal Torelli theorem, this can only be true if $\Gamma^{0}$ is a hyperelliptic curve, and $\partial_{t=0}$ is transversal to the moduli space of hyperelliptic curves. In order to finish the proof that up to the order $O\left(t^{2}\right)$ the curve $\gamma^{t}$ does not change, we shall show that if (A.6) is fulfilled for some hyperelliptic curve $\Gamma^{0}$, then the vector $\partial_{t=0}$ is tangent to the moduli space of hyperelliptic curves.

We fix on $\Gamma^{0}$ a pair of distinct points $P^{ \pm}$for which there exists a function $\lambda$ with simple poles at these points and holomorphic everywhere else. We may choose them so that $\mathrm{d} E\left(P^{ \pm}\right) \neq 0$. Let

$$
\lambda=a^{ \pm}\left(E-E_{ \pm}^{0}\right)+b^{ \pm}+O\left(E-E_{ \pm}^{0}\right), E_{ \pm}^{0}=E\left(P^{ \pm}\right)
$$

be the expansions of $\lambda$ at the points $P^{ \pm}$. Then on $\Gamma^{t}$, there exists a unique meromorphic real-normalized Abelian integral $\lambda(E, t)$, with simple poles at the points $P^{ \pm, t}$ given by $E\left(P^{ \pm, t}\right) \equiv E_{ \pm}^{0}$, and whose expansion near these points has the form of the right-hand side of (A.8) identically in $t$. Arguing just as before, we can conclude that

$$
\left.\partial_{t} \lambda(E, t)\right|_{t=0}=0 .
$$


This means that, up to order $O\left(t^{2}\right)$, the periods of $d \lambda(E, t)$ are the same as the periods of $d \lambda(E, 0)$, and thus equal to zero. The function $\lambda(t)$ is a single-valued function with only two simple poles, the curve $\Gamma^{t}$ is hyperelliptic, and $\partial_{t=0}$ is a tangent vector to the moduli space of hyperelliptic curves.

This completes the proof that, up to order $O\left(t^{2}\right)$, the curve $\Gamma^{0}=\Gamma^{t}$ does not change. Now we have to show that the punctures and the jets of local coordinates also do not change.

For $g=0$ or 1 , the nontrivial automorphism group of the curve allows us to fix the puncture $P_{1}$. For $g>1$, there exists a holomorphic differential d $\omega_{0}$ with

$$
\mathrm{d} \omega_{0}\left(P_{\alpha(0)}\right) \neq 0
$$

and $\mathrm{d} E$ not vanishing at at least one of its zeros

$$
\mathrm{d} \omega_{0}\left(p_{0}\right)=0, \mathrm{~d} E\left(p_{0}\right) \neq 0 .
$$

As before, as a consequence, the Abelian integral

$$
\omega_{0}(P)=\int_{P_{1}}^{P} \mathrm{~d} \omega_{0}
$$

must satisfy the relation

$$
\left.\partial_{t} \omega_{0}(E, t)\right|_{t=0}=0,
$$

when it is considered as a function of $E$. The derivatives with fixed $E$ or $P$ are related to each other by (c.f. (80))

$$
\partial_{t} \omega_{0}(P)=\partial_{t} \omega_{0}(E, t)+\partial_{t} E(P, t) \frac{\mathrm{d} \omega_{0}}{\mathrm{~d} E}
$$

Taking the derivative of (A.12) at $P=p_{0}$, we get

$$
0=\left.\partial_{t} \omega_{0}\left(p_{0}\right)\right|_{t=0}=-\left(\left.\partial_{t} z\left(P_{1}(t)\right)\right|_{t=0}\right) \frac{\mathrm{d} \omega_{0}}{\mathrm{~d} z}\left(P_{1}(0)\right),
$$

where $z(P)$ is any local coordinate in a neighborhood of $P_{1}(0)$. Thus, up to order $O\left(t^{2}\right)$, the point $P_{1}(t)=P_{1}(0)$ does not change.

At the points $P_{\alpha}(t)$ the function $E$ has a constant value (which is infinity). In view of (A.12) and the fact that $\left.\partial_{t} P_{1}\right|_{t=0}=0$, we then have

$$
\left.\left(\partial_{t} \int_{P_{1}}^{P_{\alpha}(t)} \mathrm{d} \omega_{0}\right)\right|_{t=0}=0 .
$$


Since $\mathrm{d} \omega_{0}$ does not vanish at $P_{\alpha(0)}$ (c.f. (A.10)), we conclude that $\left.\partial_{t} P_{\alpha}(t)\right|_{t=0}=0$.

The next step is to prove that jets of local coordinates at the punctures do not change. We proceed in the same way as before and consider the derivatives of the real-normalized differentials $\mathrm{d} \Omega_{(k)}$ which have poles of the form

$$
\mathrm{d} \Omega_{k}=\mathrm{d}\left(E^{k_{\alpha} / n_{\alpha}}+O(1)\right), \quad k=\left(k_{1}, \ldots, k_{N}\right), k_{\alpha} \leq n_{\alpha},
$$

at the punctures $P_{\alpha}$, and are holomorphic otherwise. Exactly in the same way as before, our assumptions imply that

$$
\left.\partial_{t} \Omega_{k}(E, t)\right|_{t=0}=0 .
$$

The last equalities yield that the $n_{\alpha}$-jets of local coordinates which were used to define $E$ do not change. The theorem is proved under the assumption that all zeros of $\mathrm{d} E$ on $\Gamma^{0}$ are simple.

We consider now the general case, where $\mathrm{d} E$ may have multiple zeroes. Let $D=\sum \mu_{s} \gamma_{s}$ be the zero divisor of $\mathrm{d} E$. The degree of this divisor is equal to

$$
\sum_{s} \mu_{s}=2 g-2+N+\sum_{\alpha=1}^{N} n_{\alpha} .
$$

Consider a small neighborhood of $\gamma_{s}$, viewed as a point of the fibration $\mathcal{N}$, above the original data point in the universal configuration space. Viewed as a function on the fibration, $E$ is a deformation of its value $E\left(P, m_{0}\right)$ above the original data point, with multiple critical points $\gamma_{s}$. Therefore, on each of the corresponding curve, there exists a local coordinate $w_{s}$ such that

$$
E=w_{s}^{\mu_{s}+1}(P, m)+\sum_{i=0}^{\mu_{s}-1} E_{s, i}(m) w_{s}^{i}(P, m) .
$$

The coefficients $E_{s, i}(m)$ of the polynomial (A.20) are well-defined functions on $\mathcal{M}_{g}(n, m)$. If $\mu_{s}=1$, then $E_{s, 0}$ coinsides with the critical value $E(\gamma)$ from (A.3). In the same way as before, we can prove that (A.1) implies that

$$
\left.\partial_{t} E_{s, i}(t)\right|_{t=0}=0,
$$

after which the arguments become identical to the previously considered case. Hence Theorem 1 is proved. 


\section{Acknowledgments}

The authors would like to thank E. D'Hoker, A. Gorsky, A. Marshakov, A. Mironov, and A. Morozov for very helpful conversations.

\section{References}

[1] N. Seiberg \& E. Witten, Electric-magnetic duality, monopole condensation, and confinement in N=2 supersymmetric Yang-Mills theory, Nuclear Phys. B 426 (1994) 19, hepth/9407087; Monopoles, duality, and chiral symmetry breaking in N=2 supersymmetric QCD, Nuclear Phys. B 431 (1994) 484, hepth/9408099.

[2] A. Klemm, W. Lerche, S. Yankielowicz \& S. Theisen, Simple singularities and N=2 supersymmetric Yang-Mills theory, Phys. Lett. 344 B (1995) 169;

P. C. Argyres \& A. E Faraggi, The vacuum structure and spectrum of N=2 supersymmetric $S U(N)$ gauge theory, Phys. Rev. Lett. 73 (1995) 3931, hepth/9411057;

M. R. Douglas \& S. Shenker, Dynamics of $S U(N)$ supersymmetric gauge theory, hepth/9503163;

P. C. Argyres \& M. R. Douglas, New phenomena in SU(3) supersymmetric gauge theory, hepth/9505062.

[3] A. Hanany \& Y. Oz, On the quantum moduli space of vacua of $N=2$ supersymmetric $S U(N)$ gauge theories, hepth/9505075

P. Argyres, R. Plesser \& A. Shapere, The Coulomb phase of N=2 supersymmetric QCD, Phys. Rev. Lett. 75 (1995) 1699-1702, hepth/9505100

J. Minahan \& D. Nemeshansky, Hyperelliptic curves for supersymmetric YangMills, hepth/9507032

P. C. Argyres \& A. Shapere, The vacuum structure of $N=2$ super $Q C D$ with classical gauge groups, hepth/9509175

A. Hanany, On the quantum moduli space of vacua of $N=2$ supersymmetric gauge theories, hepth/9509176.

[4] U. H. Danielsson \& B. Sundborg, Exceptional Equivalences in $N=2$ Supersymmetric Yang-Mills Theory, USITP-95-12, UUITP-20/95

The moduli space and monodromies of $N=2$ supersymmetric $S O(2 r+1)$ Yang-Mills theory, hepth/9504102;

A. Brandhuber \& K. Landsteiner, On the monodromies of $N=2$ supersymmetric Yang-Mills theory with gauge group SO(2n), hepth/9507008

[5] A. Gorsky, I. Krichever, A. Marshakov, A. Mironov \& A. Morozov, Integrability and Seiberg-Witten exact solutions, Phys. Lett. B 355 (1995) 466, hepth/9505035;

E. Martinec \& N. Warner, Integrable systems and supersymmetric gauge theory, hepth/9509161, hepth/9511052

A. Gorsky \& A. Marshakov, Towards effective topological theories on spectral curves, hepth/9510224

T. Nakatsu \& K. Takasaki, Whitham-Toda hierarchy and $N=2$ supersymmetric Yang-Mills theory, hepth/9509162 
H. Itoyama \& A. Morozov, Prepotential and the Seiberg-Witten theory, hepth/9511126, hepth/9512161, hepth/9601168

A. Marshakov, Exact solutions to quantum field theories and integrable equations, hepth/9602005

[6] R. Donagi \& E. Witten, Supersymmetric Yang-Mills theory and integrable systems, hepth/950101;

E. Martinec, Integrable structures in supersymmetric gauge and string theory, hepth/9510204

[7] H. Flaschka, M. G. Forest \& D. W. McLaughlin, Multiphase averaging and the inverse spectral solution of the $K d V$ equation, Comm. Pure Appl. Math. 33 (1980) 739-784;

B. A. Dubrovin \& S. P. Novikov, The Hamiltonian formalism for $1 \mathrm{D}$ systems of hydrodynamic type and the Bogolyubov-Whitham averaging method, Soviet Math. Dokl. 2 (1983) 665-669;

I. M. Krichever, The dispersionless Lax equation and topological minimal models, Comm. Math. Phys. 143 (1992) 415-429;

B. A. Dubrovin, Hamiltonian formalism of Whitham hierarchies and topological Landau-Ginzburg models, Comm. Math. Phys. 145 (1992) 195-207.

[8] - Elliptic solutions of the Kadomtsev-Petviashvili equation and integrable systems of particles, Functional Anal. Appl. 14 (1980) 282-290.

[9] - The averaging method for 2D systems, Functional Anal. Appl. 22 (1988) $200-212$.

[10] The $\tau$-function of the universal Whitham hierarchy, matrix models, and topological field theories, Comm. Pure Appl. Math. 47 (1994) 437-475.

[11] A. Gorsky, A. Marshakov, A. Mironov \& A. Morozov, $N=2$ supersymmetric QCD and integrable spin chains: rational case $N_{f}<2 N_{c}$, hepth/9603140

[12] I. M. Krichever, Methods of algebraic geometry in the theory of non-linear equations, Russian Math. Surveys 32 (1977) 185-213.

[13] S. P. Novikov \& A. Veselov, On Poisson brackets compatible with algebraic geometry and Korteweg-de Vries dynamics on the space of finite-zone potentials, Soviet Math. Dokl. 26 (1982) 357-362; Poisson brackets and complex tori, Proc. Steklov Inst. Math. 165 (1985) 53-65.

[14] S. P. Novikov, S. V. Manakov, L. P. Pitaevski \& V. E. Zakharov, Theory of Solitons, Contemp. Soviet Math., Plenum Press, 1984, New York;

H. Flaschka \& D. McLaughlin, Canonically conjugate variables for $K d V$ and the Toda lattice with periodic boundary conditions, Prog. Theoret. Phys. $\mathbf{5 5}$ (1976) 438-456.

[15] I. M. Gelfand \& L. A. Dickey, Fractional powers of operators and Hamiltonian systems, Functional Anal. Appl. 10 (1976) 13-29;

L. A. Dickey, Soliton equations and Hamiltonian systems, World Scientific, 1991, Singapore. 
[16] I. M. Krichever, The periodic nonabelian Toda lattice and its two-dimensional generalization, Uspekhi Mat. Nauk 36 (1981) 72-77.

[17] F. Calogero, A method to generate soluble non-linear evolution equations, Lett. Nuovo Cimento 14 (1975) 443-447;

J. Moser, Three integrable Hamiltonian systems connected with isospectral deformations, Adv. Math. 16 (1975) 197-220;

A. M. Perelomov, Completely integrable classical systems connected with semisimple Lie algebras, Lett. Math. Phys. 1 (1977) 531-540.

[18] A. Gorsky \& N. Nekrasov, Elliptic Calogero-Moser system from two-dimensional current algebra, hepth/9401021.

Landau Institute for Theoretical Physics, Moscow Columbia University, New York 